\title{
Identification of novel disease relevant genetic modifiers affecting the SHH pathway in the developing brain
}

Nora Mecklenburg ${ }^{1,10}$, Izabela Kowalczyk ${ }^{1,10}$, Franziska Witte ${ }^{2,9,10}$, Jessica Görne ${ }^{1}$, Alena Laier ${ }^{1}$, Hannes Gonschior ${ }^{3}$, Martin Lehmann ${ }^{3}$, Matthias Richter ${ }^{4}$, Anje Sporbert ${ }^{4}$, Bettina Purfürst ${ }^{5}$, Norbert Hübner $2,6,7,8$, and Annette Hammes ${ }^{1,11, *}$

${ }^{1}$ Disorders of the Nervous System, Max-Delbrück-Center for Molecular Medicine in the Helmholtz Association (MDC), 13125 Berlin, Germany

${ }^{2}$ Cardiovascular and Metabolic Sciences, Max Delbrück Center for Molecular Medicine in the Helmholtz Association (MDC), 13125 Berlin, Germany

${ }^{3}$ Cellular Imaging, Light Microscopy, Leibniz-Research Institute for Molecular Pharmacology (FMP), 13125 Berlin, Germany

${ }^{4}$ Advanced light microscopy technology platform, Max-Delbrück-Center for Molecular Medicine in the Helmholtz Association (MDC), 13125 Berlin, Germany

${ }^{5}$ Electron microscopy technology platform, Max-Delbrück-Center for Molecular Medicine in the Helmholtz Association (MDC), 13125 Berlin, Germany

${ }^{6}$ German Center for Cardiovascular Research (DZHK), Partner Site Berlin, 10785 Berlin, Germany

${ }^{7}$ Charité-Universitätsmedizin Berlin, 10117 Berlin, Germany

${ }^{8}$ Berlin Institute of Health (BIH), 10178 Berlin, Germany

${ }^{9}$ Present address: Structural Biology, Lead Discovery, Nuvisan ICB GmbH, 13353

Berlin, Germany

${ }^{10}$ These authors contributed equally

${ }^{11}$ Lead contact

*Correspondence: hammes@mdc-berlin.de 


\section{SUMMARY}

Pathogenic gene variants in humans affecting the sonic hedgehog $(\mathrm{SHH})$ pathway lead to severe brain malformations with variable penetrance due to unknown genetic modifiers. To identify such modifiers, we established novel congenic mouse models. LRP2 deficient C57BL/6N mice suffer from heart outflow tract defects and holoprosencephaly caused by impaired SHH activity. These defects are fully rescued on FVB/N background indicating a strong influence of modifier genes. Applying comparative transcriptomics, we identified Pttg1 and Ulk4 as candidate modifiers upregulated in the rescue strain. Functional analyses showed that ULK4 and PTTG1, both microtubule-associated proteins, are new positive regulators of $\mathrm{SHH}$ signaling, rendering the pathway more resilient to disturbances. In addition, we characterized PTTG1 as a novel primary cilia component in the neuroepithelium. The identification of genes, that powerfully modulate the penetrance of genetic disturbances affecting the brain and heart, is likely relevant to understand variability in human congenital disorders.

\section{KEYWORDS:}

$\mathrm{SHH}$, primary cilium, forebrain development, holoprosencephaly, heart, congenital disorder, LRP2, genetic modifiers, PTTG1 


\section{INTRODUCTION}

Holoprosencephaly (HPE) is the most common structural defect of human forebrain development resulting from impaired separation of the two cephalic hemispheres and typically accompanied by craniofacial malformations (Geng and Oliver, 2009; Hong and Krauss, 2018; Krauss, 2007; Ming and Muenke, 2002; Muenke and Beachy, 2000). HPE is a complex condition caused by genetic predisposition and exposure to environmental toxins (Hong et al., 2020; Krauss and Hong, 2016; Tekendo-Ngongang et al., 1993). The genetic contribution can be either monogenic or polygenic (Krauss and Hong, 2016; Roessler and Muenke, 2010). Single mutations causing HPE have been identified in mice and humans (Hayhurst and McConnell, 2003; Roessler and Muenke, 2010; Roessler et al., 1996). Mutations in the human sonic hedgehog (SHH) gene and its downstream effector genes account for at least $5 \%$ of autosomal dominant nonsyndromic HPE cases (Wallis and Muenke, 2000, 1999; Wallis et al., 1999). Mice deficient for SHH develop forebrain defects resembling those of human HPE cases (Chiang et al., 1996). Another gene more recently implicated in the etiology of HPE in mice and humans is LRP2 (low-density lipoprotein receptor-related protein 2) (Kim et al., 2019; Rosenfeld et al., 2010; Spoelgen et al., 2005; Willnow et al., 1996). Loss of LRP2 activity in $\mathrm{Lrp}^{-/}$mouse embryos causes HPE, associated with severe craniofacial defects (Spoelgen et al., 2005; Willnow et al., 1996). LRP2 is also a component of the SHH signaling machinery in the primary cilium (Christ et al., 2012), as LRP2 deficiency leads to an impairment in the response of the ventral forebrain neuroepithelium to $\mathrm{SHH}$.

Humans with autosomal recessive $L R P 2$ gene defects suffer from Donnai-Barrow syndrome with clinical characteristics resembling those in the mouse model, including craniofacial anomalies, forebrain defects (Kantarci et al., 2007; Ozdemir et al., 2019), microforms of HPE (Rosenfeld et al., 2010), and heart anomalies (Pober et al., 2009). A recent publication describes severe forms of HPE in families presenting oligogenic 
events involving LRP2 (Kim et al., 2019). Inter- and intrafamilial phenotypic variability is observed in cases of the Donnai-Barrow syndrome (Khalifa et al., 2015; Longoni et al., 1993; Pober et al., 2009) and is a characteristic feature of HPE (Dubourg et al., 2018; Solomon et al., 2010). Even within pedigrees, HPE phenotypes amongst relatives, carrying the same $S H H$ mutation, can range from alobar HPE, facial abnormalities typical of HPE, to asymptomatic appearance of the carrier. Intrafamilial variability of HPE phenotypes could be due to environmental or genetic factors. (Heussler et al., 2002; Hong and Krauss, 2018; Ming and Muenke, 2002; Muenke and Beachy, 2000; Muenke and Cohen, 2000; Roessler et al., 1996).

Studies on mouse models for HPE suggest that loss of function mutations in genes relevant for forebrain development result in brain and craniofacial anomalies that recapitulate HPE phenotypes in patients carrying mutations in the same genes (Geng and Oliver, 2009; Hayhurst and McConnell, 2003; Heyne et al., 2016; Hong and Krauss, 2018). Intriguingly, the variability of the HPE expression is also reflected in gene targeted mouse models since the penetrance of the phenotype often depends on the mouse strain that is analyzed (Anderson et al., 2002; Cole and Krauss, 2003; Geng and Oliver, 2009; Geng et al., 2008; Hong and Krauss, 2018; Petryk et al., 2004; Schachter and Krauss, 2008). Thus, the mouse is an ideal model, not only to investigate the complex etiology of human HPE but also to identify disease relevant genetic modifiers. 


\section{RESULTS}

\section{Genetic background determines penetrance of brain, craniofacial and heart defects in Lrp2 $^{-/}$mutant mice}

$\mathrm{Lrp2}^{-/-}$mice on a C57BL/6N background at embryonic stage (E) 18.5 displayed severe HPE associated skull and facial dysgenesis (Figure 1A: $d-f$ ). In particular, they showed a shortened snout (Figure 1A: d), cleft lip (Figure 1A: e, arrow) and incompletely developed or even bilaterally or unilaterally absent eyes (Figure 1A: $d$ and e). Further characteristic features seen in these mutants were a shortened skull (Figure 1A: f) and an open anterior suture (Figure 1A: f, arrowhead). In contrast, Lrp2 - congenic mice with a FVB/N background showed $100 \%$ penetrant rescue of all the above described craniofacial defects (Figure 1A: j - I). HPE in Lrp2 ${ }^{-1-}$ C57BL/6N embryos was fully penetrant and characterized by a fusion of the cortical hemispheres (Figure 1B: d, arrowhead) with a single lateral ventricle (Figure 1B: $f$, arrowhead) and absent olfactory bulbs (Figure 1B: $d$ and e, asterisk). In contrast, HPE was fully rescued in all $\mathrm{Lrp}^{-/} \mathrm{FVB} / \mathrm{N}$ mutants, which presented with correctly separated cortical hemispheres, olfactory bulbs and clearly defined lateral ventricles (Figure 1B: j - I). Corpus callosum and the anterior commissure were also present in the LRP2 deficient mice on a FVB/N background (Figure 1B: I).

LRP2 deficient mice also suffer from cardiovascular anomalies including a common arterial trunk (CAT) as one of the most penetrant phenotypes on a C57BL/6N background (Baardman et al., 2016; Christ et al., 2020; Li et al., 2015). Interestingly, there is comorbidity of HPE and congenital heart disease in patients with variants of known HPE-related genes (Tekendo-Ngongang et al., 2020). Lrp2 ${ }^{-/}$embryos with CAT, also known as persistent truncus arteriosus, displayed a single common coronary outflow artery vessel instead of pulmonary artery and ascending aorta. Less frequently $\mathrm{Lrp}^{-/}$embryos displayed a double outlet right ventricle (DORV), in which the pulmonary artery and ascending aorta both connect to the right ventricle. We found 
that 15 out of $16 \mathrm{Lrp2}^{-/}$mutants on a C57BL/6N background displayed CAT above the right ventricle (Figure 1C: c, d, f, f' and Supplementary Table 1), and one remaining mutant embryo displayed DORV (Supplementary Table 1). The CAT phenotype was also visualized by pigment injection, (Figure 1C: $d$, arrowhead) indicating CAT and a ventricular septum defect. We never observed CAT in $L r p 2^{-/} \mathrm{FVB} / \mathrm{N}$ mutants; $85 \%$ of $\mathrm{Lrp2}^{-/ \mathrm{FVB}} \mathrm{N}$ embryos showed no apparent heart anomalies and a normal ventricular septum compared to control littermates (Figure 1C: i, j, I, l' and Supplementary Table

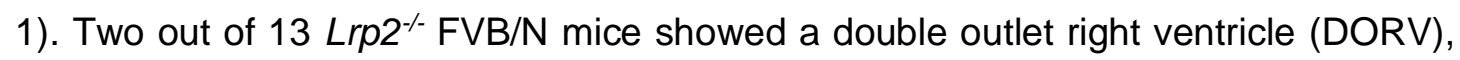
(Supplementary Table 1), which has been described in mutants on C57BL/6N strain with a similar frequency (Baardman et al., 2016). We conclude that the FVB/N background fully rescues the CAT phenotype, whereas the etiology of DORV is not influenced by the strain background, suggesting the likelihood of divergent pathogenic mechanisms for CAT and DORV in Lrp2 $2^{-/}$mutants. 


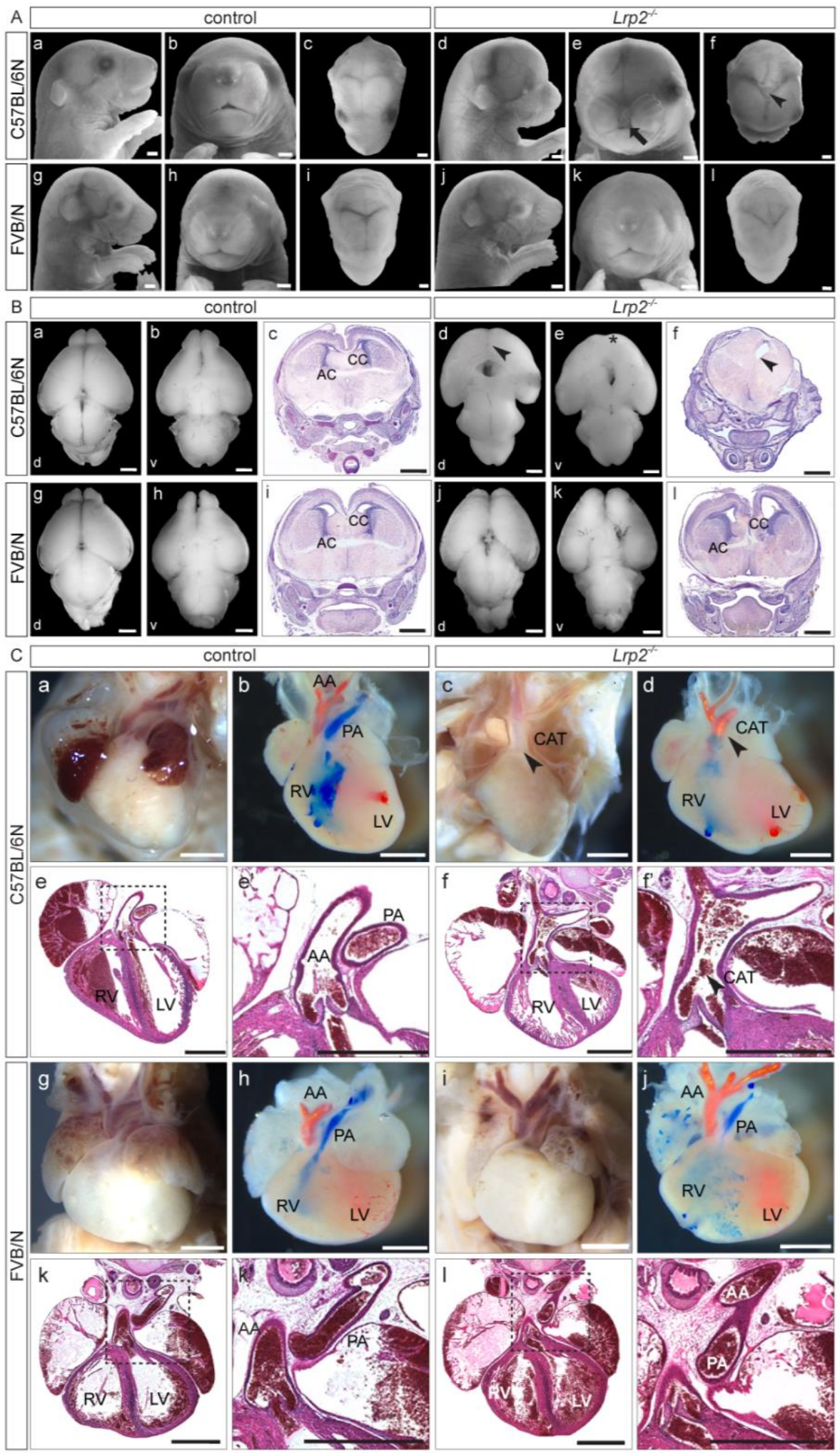

Figure 1: Rescue of craniofacial malformations, HPE and heart defects in Lrp2-FVB/N mice

(A) Lrp2 ${ }^{-1}$ C57BL/6N E18.5 embryos ( $\mathrm{n}=30$ ) with craniofacial defects: shortened snout (d), underdeveloped or missing eyes (e), cleft lip (e, arrow) and hypertelorism (e). Dorsal view on the head revealed shortened and widened skull with impaired suture formation 
and an open fontanelle (f, arrowhead). Lrp2 $2^{++}$and $L r p 2^{+/-}$littermates (a - c) served as controls $(n=66)$. Lrp2 ${ }^{-/}$FVB/N mutants $(n=28)$ displayed normal skull formation $(j)$, normal eyes and normal midface structures $(\mathbf{k})$ comparable to controls $(n=94)(\mathbf{g}-\mathbf{i})$. Note, missing pigmentation of retina due to albinism in FVB/N. Scale bars: $1 \mathrm{~mm}$

(B) Brains from E18.5 control and Lrp2\% embryos in dorsal and ventral view and NISSL stained coronal sections. Lrp2 ${ }^{-1-}$ C57BL/6N mice with HPE (d - f). Lrp2 ${ }^{-1}$ C57BL/6N brain with alobar HPE (d, arrowhead) $\left(\operatorname{Lrp2}^{-}-\mathrm{n}=4\right.$; controls $\left.\mathrm{n}=6\right)$. Olfactory bulbs were absent in mutants (e, asterisk). All sectioned Lrp2 ${ }^{-/}$C57BL/6N brains $(n=13)$ showed alobar HPE with a single lateral ventricle (f, arrowhead) compared to controls $(\mathbf{c}),(n=6)$. Missing corpus callosum (CC) and anterior commissure (AC) in $L r p 2^{-/}$C57BL/6N mice (f). Lrp2 $2^{-1}$ FVB/N embryos showed normal forebrain separation ( $\mathbf{j}-\mathbf{I}) ;\left(\operatorname{Lrp2^{-/}} n=5\right.$; controls $\left.n=3\right)$. Olfactory bulbs were present in $L r p 2^{-/-F V B / N}$ brains $(\mathbf{k})$. All $L r p 2^{-/-F V B / N}$ sectioned brains $(n=6)$ displayed normally separated ventricles, normal CC and AC (I) comparable to controls $(\mathbf{i}),(n=4)$. Scale bars: $1 \mathrm{~mm}$. d: dorsal, v: ventral.

(C) Analysis of heart outflow tract phenotype in $\mathrm{Lrp2}^{-/-}$mutants and controls.

C57BL/6N (a and $\mathbf{b}$ ) and FVB/N (g and $\mathbf{h}$ ) control (refers to $L r p 2^{+/+}$and $L r p 2^{+/-}$) hearts at E18.5 by external inspection and pigment injection.

In controls ( $\mathbf{b}$ and $\mathbf{h}$ ) injection of Batson's red pigment into the left ventricle after cutting the ductus arteriosus indicated the ascending aorta in red (AA). Injection of blue pigment into the right ventricle labeled the pulmonary artery in blue (PA).

Frontal plane H\&E stained paraffin sections through hearts of control embryos (e, e' and $\mathbf{k}, \mathbf{k}^{\prime}$ ) depicted normally separated ascending aorta (AA) and pulmonary artery (PA).

Lrp2 $2^{-/}$mutants (15/16) on C57BL/6N background suffered from common arterial trunk (CAT), (c, arrowhead). 1/16 mutants suffered from double outlet right ventricle (DORV, see

\section{Supplementary Table 1).}

Injection of the red pigment into the mutant's left ventricle followed by injection of blue ink into the right ventricle showed both pigments in the CAT and indicated an additional ventricular septum defect (d, arrowhead). CAT in $\mathrm{Lrp2}^{-/}$C57BL/6N embryos was also shown on H\&E stained frontal heart sections (f, f')

No CAT was detected in $L r p 2^{--}$mutants on FVB/N background (i, j, I, l'). $85 \%$ of $L r p 2^{-1-}$ FVB/N embryos displayed a normal heart morphology with a proper connection of the ascending aorta to the left ventricle and of the pulmonary artery to the right ventricle. 2 out of $13 \mathrm{Lpr}^{-1-}$ FVB/N mice showed DORV (Supplementary Table 1).

Scale bars: $1 \mathrm{~mm}$. LV: left ventricle; RV: right ventricle. 


\section{Rescue of SHH activity in neuroepithelial stem cells}

In the neuroepithelium, LRP2 is located at the base of the primary cilium, where it teams up with Patched1 to mediate endocytic uptake of SHH and most importantly recycling of the morphogen (Christ et al., 2012). Consistent with our previous findings, Lrp2 $^{-1}$ C57BL/6N embryos showed normal SHH pattern in the prechordal plate, but little to no SHH in the overlying neuroepithelial stem cells compared to control embryos during the critical stages of forebrain specification at the 8 somite stage (Figure 2B). $\mathrm{SHH}$ protein was detected later in the neuroepithelium at the 11 somite stage, which is too late for proper ventral midline specification (Figure $2 \mathrm{~B}$ ). Intriguingly, all $\mathrm{Lrp2}^{-1}$ FVB/N mutants showed completely normal $\mathrm{SHH}$ localization throughout all critical developmental stages (Figure 2B). To exclude later onset defects, we analyzed later embryonic stages. A fully penetrant hallmark of the $L r p 2^{-/}$C57BL/6N embryos at E10.5 is the lack of Shh RNA and protein in the telencephalon (Christ et al., 2012; Spoelgen et al., 2005) (Figures 2C, arrowheads and S2A: b) and in the ventral midline of the diencephalon (Figure S2B: b). In contrast, Lrp2 ${ }^{-/}$FVB/N mutants showed normal Shh RNA expression and $\mathrm{SHH}$ protein localization in the ventral telencephalon and diencephalon (Figures 2C, arrowhead, S2A: d, and S2B: d).

Normal Nkx2.1 expression pattern comparable to controls (Figure S2C: a, b, e, f) confirmed proper activation of SHH downstream targets and intact ventral forebrain patterning in $\mathrm{Lrp2}^{-/} \mathrm{FVB} / \mathrm{N}$ mice (Figure S2C: $\mathrm{g}, \mathrm{h}$ ) in contrast to mutants on a C57BL/6N background, which lacked $N k x 2.1$ expression in the ventral forebrain (Figure S2C: $c, d$ ).

Thus, defects in the SHH pathway caused by loss of LRP2 were fully rescued on a FVB/N background.

Consequently, the severe telencephalic vesicle separation defects in $\mathrm{Lrp2} 2^{-/ \mathrm{C} 57 \mathrm{BL} / 6 \mathrm{~N}}$ mutants (Figure S2D: $\mathrm{c}, \mathrm{d}$ ) did not manifest in mutants on a FVB/N background displaying properly separated forebrain hemispheres at E12.5 (Figure S2D: g, h). 


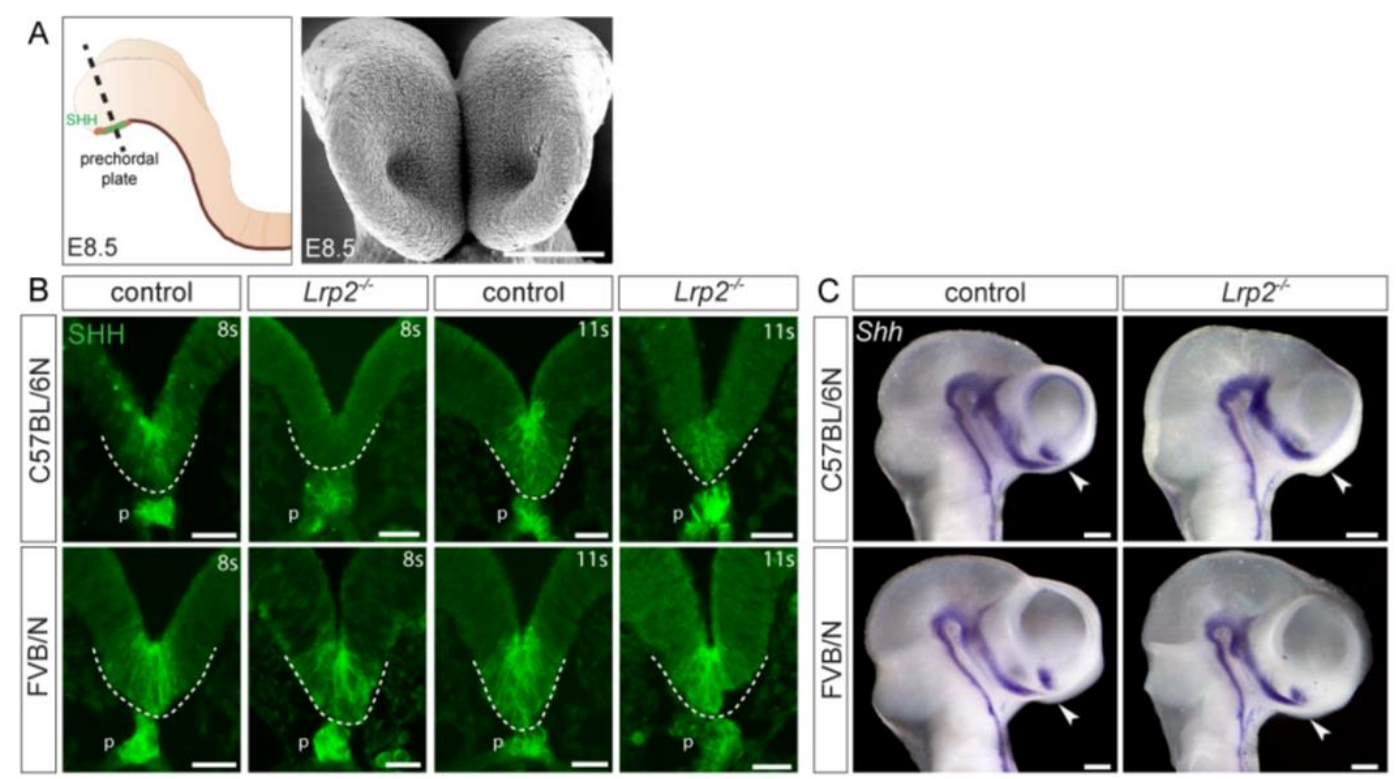

Figure 2: Loss of SHH is rescued in the ventral forebrain of $\mathrm{Lrp2}^{-/-F V B / N}$ mutants

(A) Schematic of E8.5 mouse embryo indicating the prechordal plate, underlying the neuroepithelium. Dotted line indicates section planes shown in (B). Scanning electron microscopy image represents the front view on the developing neural folds. Scale bar: 100 $\mu \mathrm{m}$.

(B) Immunohistology for SHH on coronal sections of anterior neural folds in control ( $\mathrm{Lrp2}^{+/+}$ and $\left.L r p 2^{+/}\right)$and $L r p 2^{-/}$embryos on C57BL/6N and FVB/N background.

In 8 somite stage ( $8 \mathrm{~s}$ ) control embryos $(\mathrm{n}=7)$, SHH was detected in the prechordal plate and in the neuroepithelium (above the dotted line). Lrp2- C57BL/6N embryos at $8 \mathrm{~s}(\mathrm{n}=$ 4), showed SHH in the prechordal plate, but little to none $\mathrm{SHH}$ in the neuroepithelium. In 11 somite stage embryos $\mathrm{SHH}$ appeared in the neuroepithelium of mutant embryos $(n=3$; $\mathrm{n}=4$ for controls). Lrp2 ${ }^{-1-F V B / N}$ embryos $(\mathrm{n}=8$ at $8-9 \mathrm{~s}, \mathrm{n}=2$ at $11 \mathrm{~s})$ displayed normal $\mathrm{SHH}$ pattern in the neuroepithelium throughout development, comparable to somite matched control samples ( $n=10$ at $8-9 \mathrm{~s}, \mathrm{n}=8$ at $11 \mathrm{~s}$ ). Scale bars: $25 \mu \mathrm{m}$. p: prechordal plate, s: somites.

(C) Whole mount ISH hybridization for Shh on E10.5 embryonic heads. Controls $(n=5)$ showed typical Shh expression pattern with a prominent signal in the ventral telencephalon (arrowhead). Lrp2 ${ }^{-1}$ C57BL/6N somite matched embryos $(\mathrm{n}=9)$ had no telencephalic Shh signal (arrowhead). Lrp2 ${ }^{-1-} \mathrm{FVB} / \mathrm{N}$ embryos $(\mathrm{n}=8)$ displayed the same expression pattern as controls $(n=4)$. Scale bars: $250 \mu \mathrm{m}$.

See also Figure S2. 


\section{RNA sequencing reveals a dominant effect of $\mathrm{FVB} / \mathrm{N}$ specific rescue modifier} genes

We next analyzed the transcriptome of $\mathrm{Lrp2}^{+/+}$and $\mathrm{Lrp}^{-/-}$embryonic heads from C57BL/6N and FVB/N backgrounds (Figure 3A). To test a possible dominant effect of $\mathrm{FVB} / \mathrm{N}$ specific modifier genes, we also collected $\mathrm{Lrp2}^{+/+}$and $\mathrm{Lrp2}^{-/-}$first generation hybrids from $\mathrm{Lrp2}^{+/-} \mathrm{C} 57 \mathrm{BL} / 6 \mathrm{~N} \times \mathrm{Lrp2}^{+/-} \mathrm{FVB} / \mathrm{N}$ parents, hereon referred to as $\mathrm{F} 1$ samples, for RNA sequencing (Figure 3A). Performing DESeq2 differential expression analyses on all transcriptomes (Figure 3B heatmap and Supplementary Table 2), we detected 2170 differentially expressed genes (DEGs) between $\mathrm{Lrp2}^{-/-}$and $\mathrm{Lrp}^{+/+}$ embryos on a C57BL/6N background and only 367 DEGs for the FVB/N background and a similarly low number (241 DEGs) for the F1 background (Figure 3C). Expression levels of HPE- and SHH pathway-related transcription factors like Vax1, Six6 and Six3 were significantly decreased in $\mathrm{Lrp}^{-/} \mathrm{C} 57 \mathrm{BL} / 6 \mathrm{~N}$ mutants compared to controls, but were unchanged in $\mathrm{Lrp2}^{-/} \mathrm{FVB} / \mathrm{N}$ mutants compared to FVB/N controls (Figure 3D). Interestingly, also the $\mathrm{Lrp}^{-/} \mathrm{F} 1$ mutants showed normal expression levels for Vax1, Six6 and Six3, comparable to the $L r p 2^{+/+}$controls (Figure 3D). Detailed phenotypic analyses of the $\mathrm{Lrp}^{-1-} \mathrm{F} 1$ embryos indeed revealed a full rescue of brain and craniofacial defects in these F1 mutants (Figures 3E and S3A: e - h). They neither suffered from HPE (Figures 3E and S3A: h) nor cleft lip (Figure S3A: f). Analyzing the heart, we never observed a common outflow tract $(0 / 27)$ and $56 \%$ of the $L r p 2^{-/-}$mutant F1 embryos had normal hearts and outflow tracts (Figure S3B: $d$ - $f$ and Supplementary Table 1). However, in $44 \%$ of mutants we detected a double outlet right ventricle (DORV), where pulmonary artery and ascending aorta both connect to the right ventricle (Figure S3B: $g$ - i). Most importantly, as observed for the $\mathrm{Lrp2}^{-/} \mathrm{FVB} / \mathrm{N}$ embryos, all $L r p 2^{-/} F 1$ embryos (8/8) showed normal Shh expression in the developing forebrain similar to control embryos (Figure 3F, arrowheads).

Since none (0/68) of the hybrid C57BL/6N; FVB/N Lrp2 $2^{-/}$F1 mutants suffered from HPE and SHH activity was fully rescued in $\mathrm{Lrp}^{-/-} \mathrm{F} 1$ embryos, we concluded that 
FVB/N allele-specific expression of yet unidentified genes has a dominant rescue effect. We hypothesize that such factors predispose neuroepithelial stem cells in FVB/N and F1 mice to maintain sufficient SHH activity and signaling despite the loss of LRP2 and thereby prevent HPE (Figure 3G).
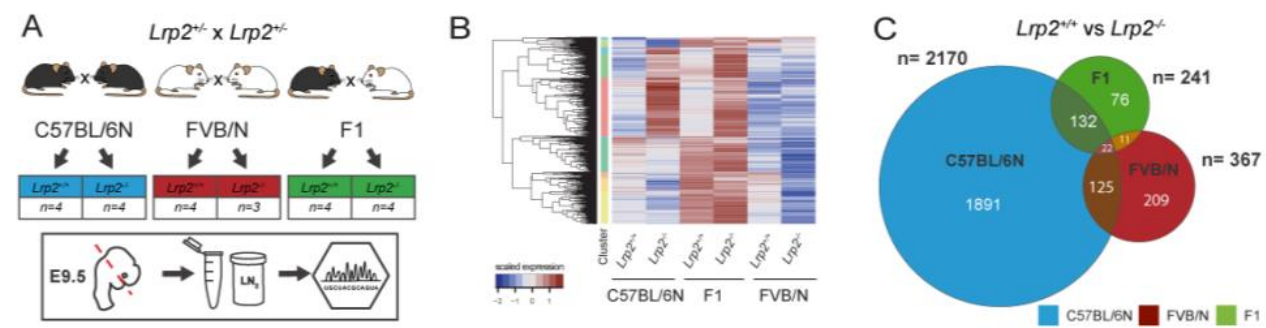

D
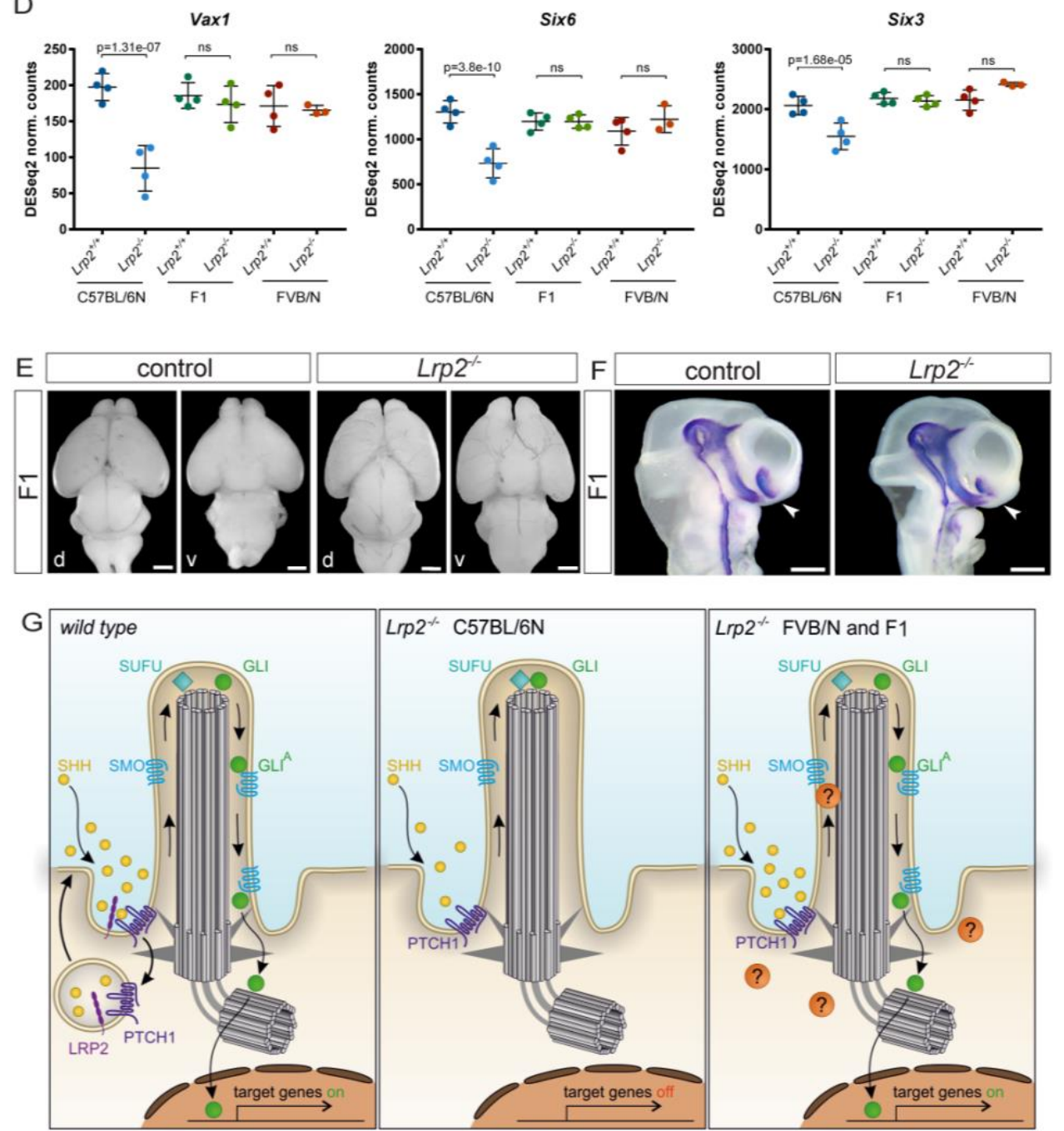

Figure 3: Dominant effect of FVB/N specific rescue modifier genes

(A) Collection of 24 somites staged $\mathrm{Lrp2}^{+/+}$and $\mathrm{Lrp2}^{-/-}$embryonic heads for RNA deep sequencing from C57BL/6N, FVB/N and F1 background. 
(B) Heatmap for RNA sequencing results.

(C) Venn diagram with numbers of DEGs comparing $L r p 2^{+/+}$and $L r p 2^{-1-}$ samples on $\mathrm{C} 57 \mathrm{BL} / 6 \mathrm{~N}, \mathrm{FVB} / \mathrm{N}$ and $\mathrm{F} 1$ background. Illustrated are the numbers of distinct and overlapping DEGs.

(D) DESeq2 normalized counts for Vax1, Six6, and Six3.

(E) Brains from E18.5 control (refers to $L r p 2^{+/+}$and $\left.L r p 2^{+-}\right)(\mathrm{n}=17)$ and $L r p 2^{-/} \mathrm{F} 1$ embryos $(\mathrm{n}=19)$ in dorsal and ventral view. $L r p 2^{-/} \mathrm{F} 1$ mice never displayed HPE $(0 / 19)$ and showed normal separation of the forebrain hemispheres. Olfactory bulbs were present in $L r p 2^{-/ F 1}$ brains. Scale bars: $1 \mathrm{~mm}$. d: dorsal, v: ventral.

(F) Whole mount ISH hybridization for Shh on E10.5 embryonic heads. Both, controls $\left(L r p 2^{+/+}\right.$and $\left.L r p 2^{+/}\right)(\mathrm{n}=6)$ and $L r p 2^{-/-} \mathrm{F} 1$ embryos $(\mathrm{n}=8)$ showed a typical Shh pattern including a prominent signal in the ventral telencephalon (arrowheads). Scale bars: 250 $\mu \mathrm{m}$.

(G) MODEL: Genetic modifiers rendering FVB/N and F1 more resilient to disturbances in the SHH pathway.

Wild type: LRP2, a co-receptor for PTCH1, facilitates the internalization of SHH morphogen to activate the pathway. $\mathrm{Lrp2} 2^{-1} \mathrm{C} 57 \mathrm{BL} / 6 \mathrm{~N}$ : SHH internalization and signaling are impaired. $\mathrm{Lrp}^{-/} \mathrm{FVB} / \mathrm{N}$ and $\mathrm{F} 1$ : intact SHH pathway despite loss of LRP2, suggesting the presence of genetic modifiers.

See also Figure S3.

\section{Identification of strain specific modifier genes involved in ciliogenesis and SHH pathway regulation}

To identify strain specific modifier genes and pathways underlying HPE susceptibility in $\mathrm{C} 57 \mathrm{BL} / 6 \mathrm{~N}$, and $\mathrm{HPE}$ rescue in $\mathrm{FVB} / \mathrm{N}$ and $\mathrm{F} 1$, we used the transcriptome data set described above (Supplementary Table 2). However, in this approach we focused on the strain specific transcriptome differences. Thus, we compared gene expression between mutant $L r p 2^{-/-}$samples on C57BL/6N, FVB/N and F1 backgrounds (Figure 4A - 4E) and most importantly between wild type ( $\left.\mathrm{Lrp}^{+/+}\right)$samples comparing C57BL/6N, FVB/N and F1 backgrounds (Figure 4F - 4J). Venn diagrams show the number of DEGs in Lrp2 ${ }^{-/}$embryos comparing the different backgrounds (Figure $4 \mathrm{C}$ ) and in wild type $\mathrm{Lrp2}^{+/+}$embryos accordingly (Figure $4 \mathrm{H}$ ). Given the dominant effect of the FVB/N 
background, we focused on DEGs, that were identified in both comparisons, C57BL/6N versus $\mathrm{FVB} / \mathrm{N}$ as well as $\mathrm{C} 57 \mathrm{BL} / 6 \mathrm{~N}$ versus $\mathrm{F} 1$ (Figure $4 \mathrm{D}$ and $4 \mathrm{I}$ and Supplementary Table 2). Importantly, out of the 1044 DEGs from the analysis comparing $\mathrm{Lrp}^{-/}$mutant samples, 426 DEGs were also identified in the wild type $\mathrm{Lrp2}^{+/+}$comparisons (Supplementary Table 2). Thus, these DEGs, common between mutant and wild type comparisons of different strains, were not a consequence of LRP2 loss of function, but truly strain specific and therefore met our criteria of strain specific modifier candidates that can modulate signaling pathways in the wild type situation.

Since there was a clear effect on the SHH pathway in the ventral forebrain organizer we next filtered those DEGs with a known function in the SHH pathway, cilia or ciliopathies from our strain specific data sets (Figure 4D and 4I and Supplementary Table 3). Hedgehog and cilia/ciliopathy relevant genes were selected according to published data (Breslow et al., 2018; Pusapati et al., 2018) which includes the Syscilia gold standard, and their expression was visualized by volcano plots (Figure 4E and 4J and Supplementary Table 3).

We determined that among cilia- and $\mathrm{SHH}$-related genes 12 were down-regulated and 10 up-regulated in $L r p 2^{-/} \mathrm{FVB} / \mathrm{N}$ and $\mathrm{F} 1$ samples compared to $\mathrm{C} 57 \mathrm{BL} / 6 \mathrm{~N}$ (Figure 4E). In the wild type $\mathrm{Lrp}^{+/+}$comparison, we identified 20 differentially expressed cilia- and $\mathrm{SHH}$-relevant genes, of which 11 were up- and 9 down-regulated in wild type FVB/N and F1 embryos compared to C57BL/6N (Figure 4J).

For functional analyses we selected top hits, based on the highest fold change and $p$ value identified in both, the wild type and mutant, comparisons (Figure 4E and 4J). Tubulin alpha 1C (Tuba1c) and Unc-51-like kinase 4 (Ulk4) were highly differentially expressed in a strain dependent manner.

Tuba1c, a component of tubulin, was expressed at five times lower levels in FVB/N $\mathrm{Lrp2}^{-/}$and wild type $\mathrm{Lrp}^{+/+}$embryonic heads compared to both C57BL/6N genotypes. F1 samples showed also significantly lower expression levels than samples from the 
C57BL/6N strain (Figure S5A). Tuba1c is listed in the database of ciliary genes http://www.syscilia.org/goldstandard.shtml (van Dam et al., 2013; Pusapati et al., 2018), but data on its direct connection to the SHH pathway are very limited. However, pathway analysis has linked Tuba1c to the SHH "off" state (Fabregat et al., 2018; Rothfels, 2014).

Ulk4, which belongs to a family of serine/threonine kinases, has been shown to regulate acetylation of $\alpha$-tubulin, an important post-translational modification of microtubules (Lang et al., 2016). Ulk4 was 5 and 2 times higher expressed in FVB/N and F1 genotypes, respectively compared to C57BL/6N samples (Figure S5B). 


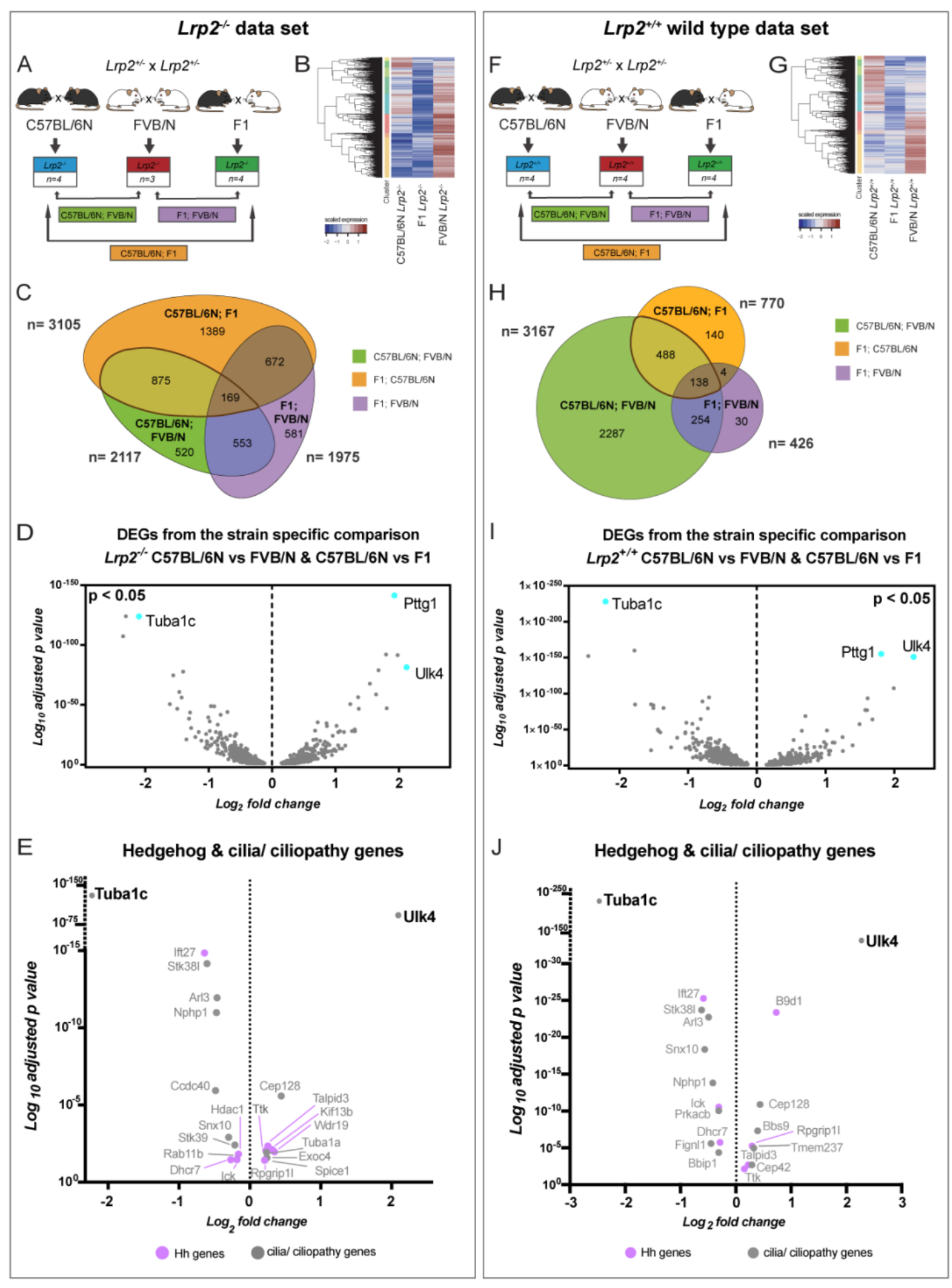

Figure 4: Comparative transcriptome analysis identifies strain-specific expression of genes involved in ciliogenesis and SHH pathway regulation

(A) and (F) RNA deep sequencing analysis on $\mathrm{Lrp2}^{--}(\mathbf{A})$ and $\mathrm{Lrp2}^{+/+}(\mathbf{F})$ embryonic heads on C57BL/6N, FVB/N, and F1 background. Comparison of the following expression profiles: $\mathrm{C} 57 \mathrm{BL} / 6 \mathrm{~N}$ versus $\mathrm{FVB/N}, \mathrm{F} 1$ versus $\mathrm{FVB} / \mathrm{N}$ and $\mathrm{C57BL} / 6 \mathrm{~N}$ versus $\mathrm{F} 1$.

(B) and (G) Pattern of the $L r p 2^{-/-}$and $L r p 2^{+/+}$heat maps for all different strains. 
(C) and (H) Venn Diagram (non-scaled) demonstrates the number of DEGs comparing Lrp2 $2^{-/}$samples $(\mathbf{C})$ and $L r p 2^{+/+}$samples $(\mathbf{H})$. C57BL/6N versus FVB/N, F1 versus FVB/N and $\mathrm{C} 57 \mathrm{BL} / 6 \mathrm{~N}$ versus $\mathrm{F} 1$ comparisons are demonstrated (colored ellipses and circles, respectively). Overlaps in two or three sets are indicated by the overlapping circles and by different color code.

(D) and (I) Volcano plots showing DEGs for $L r p 2^{-/}$samples (D) and $L r p 2^{+/+}$samples (I) that were identified in both comparisons, $\mathrm{C} 57 \mathrm{BL} / 6 \mathrm{~N}$ versus $\mathrm{FVB} / \mathrm{N}$ as well as $\mathrm{C} 57 \mathrm{BL} / 6 \mathrm{~N}$ versus F1 hybrids. (D) 562 genes were significantly down- and 482 genes significantly up-

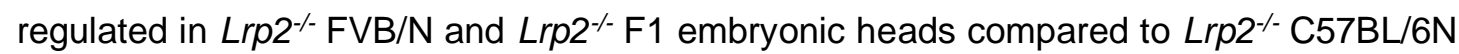
samples. (I) 342 genes were significantly down- and 284 genes significantly up-regulated in $L r p 2^{+/+} \mathrm{FVB} / \mathrm{N}$ and $\mathrm{F} 1$ samples compared to $\mathrm{C} 57 \mathrm{BL} / 6 \mathrm{~N}$ heads.

(E) and (J) Cilia, ciliopathy and hedgehog signaling pathway related genes after filtering DEGs from (D) and (I), shown in volcano plots for $\mathrm{Lrp2}^{-/-}(\mathbf{E})$ and $\mathrm{Lrp2}^{+/+}$samples (J). Note, some of the cilia/ciliopathy genes are also hedgehog related genes and therefore labeled in purple.

\section{Ulk4 increases cellular SHH signaling capacity}

We next analyzed the modifier genes in a context independent of LRP2 loss of function.

To test whether Tuba1c and Ulk4 expression has functional effects on the SHH pathway we used a dual luciferase reporter assay (Christ et al., 2012; Sasaki et al., 1997; Taipale et al., 2000; Zhang et al., 2006) and quantified activity of SHH signaling upon expression of modifier candidates. Expression of B9d1, a $\mathrm{SHH}$ signaling modulator, was used as a positive control (Chih et al., 2012; Garcia-Gonzalo et al., 2011; Gerhardt et al., 2016). Overexpression of B9d1 and Ulk4, respectively in transfected NIH-3T3 cells treated with SHH-Np resulted in a significant induction of $\mathrm{SHH}$ responsive and GLI driven relative luciferase levels compared to controls (Figure 5A). The induction was inhibited by KAAD-cyclopamine, showing a specific effect on the canonical SHH pathway. In contrast, cells transfected with Tuba1c showed no induction of GLI based luciferase activity after SHH stimulation compared to controls (Figure 5A). 
We conclude that Ulk4, which is highly expressed in the $\mathrm{FVB} / \mathrm{N}$ and $\mathrm{F} 1$ rescue backgrounds, has a promoting effect on $\mathrm{SHH}$ signaling efficiency and represents a new SHH pathway modifier gene.

\section{Identification of PTTG1 as a novel SHH pathway modifier}

We next focused on highly regulated genes that had not hitherto been associated with the primary cilium or SHH pathway and went back to the unbiased set of DEGs from all strain comparisons. The transcript with the highest fold change value and most significant $p$-value was pituitary tumor transforming gene 1(Pttg1), also known as securin (Figure 4D and 4I). Higher Pttg1 transcript levels (Figure S5C) found in rescue backgrounds were reflected at the protein level. Western analysis detected significantly higher amounts of PTTG1 protein in FVB/N and F1 total embryos compared to C57BL/6N samples (Figure 5B). In addition, we demonstrated significantly higher immunofluorescence intensities for PTTG1 in the FVB/N and F1 forebrain neuroepithelium compared to C57BL/6N (Figure S5D).

We tested whether Pttg1 can influence SHH signaling capacity. Pttg1 overexpression in NIH-3T3 cells resulted in a significantly higher activation of the GLI luciferase reporter after SHH stimulation, compared to control vector transfection, and this was inhibited by KAAD-cyclopamine (Figure 5A). Our DESeq2 data and quantitative RTPCR experiments showed a two-fold increase in Gli1 levels in FVB/N rescue (Figure S5E and S5F) supporting the hypothesis that higher Pttg1 expression levels could be one of the causative factors for more efficient $\mathrm{SHH}$ signaling. 


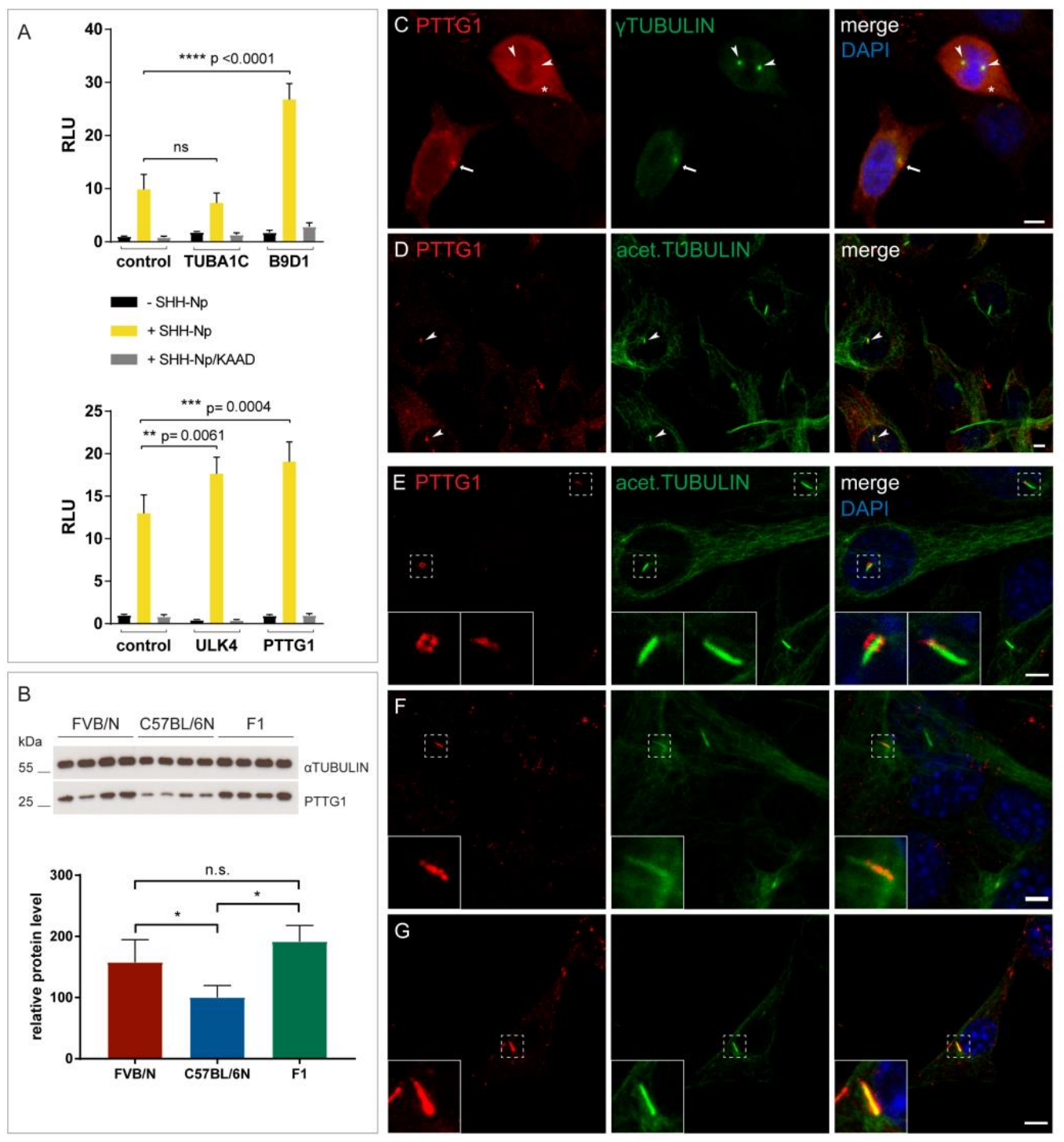

Figure 5: Novel SHH pathway modifier genes

(A) Luciferase reporter assay on NIH-3T3 cells. Significant induction of relative luciferase levels compared to controls, upon overexpression of B9d1, Ulk4, and Pttg1. Tuba1c expression showed no induction of $\mathrm{SHH}$ signaling. $\mathrm{NIH}-3 \mathrm{~T} 3$ cells were treated with medium containing SHH-Np or control medium. KAAD-cyclopamine was used to confirm the canonical SHH pathway response. Significance assessed by two-way ANOVA; ${ }^{\star \star} p<$ $0.001,{ }^{* *} \mathrm{p}<0.0001$, ns: not significant; RLU: relative light units.

(B) Western blot analysis of total PTTG1 protein levels in FVB/N, C57BL/6N, and F1 E8.5 Lrp2 ${ }^{+/+}$embryos (13 somites, $n=4$ for each background). PTTG1 signals, normalized to $\alpha$ tubulin, showed significantly higher PTTG1 levels in FVB/N and F1 compared to C57BL/6N embryos. Bar graph shows the quantification with significance assessed by un-paired ttest; * $\mathrm{p}<0.05$, ns: not significant.

(C) - (G) Subcellular localization of PTTG1 in NIH-3T3 cells by immunocytochemistry.

(C) Confocal microscopy detected PTTG1 in mitotic cells at the perinuclear region, 
concentrated at the centrosome and centrosomes (C, arrow and arrowheads) positive for $\mathrm{Y}$-tubulin and in the cytoplasm (C, asterisk).

(D) Interphase/quiescent cells showed PTTG1 localized to a subset of primary cilia stained with acetylated tubulin (D, arrowheads). Experiments were repeated at least five times in triplicates. Scale bars: $1 \mu \mathrm{m}$.

(E) - (G) PTTG1 localized to a subset of primary cilia in a heterogeneous pattern. PTTG1 was detected in the periciliary region $(\mathbf{E})$ and along the ciliary shaft, either at the proximal part $(\mathbf{F})$ or covering entire cilium $(\mathbf{G})$. Insets show the magnification of the chosen cilia (dashed line squares). Experiments were repeated at least five times in triplicates. Scale bars: $1 \mu \mathrm{m}$.

See also Figure S5.

\section{PTTG1 is a novel ciliary protein in the brain}

We next analyzed the subcellular localization of PTTG1 and as expected (MorenoMateos et al., 2011; Tong et al., 2008) we detected PTTG1 in the perinuclear region concentrated at the centrosome and centrosomes of mitotic NIH-3T3 cells (Figures 5C, arrow and arrowheads respectively, and S5G: a). In some cells we also observed a dispersed PTTG1 signal in the cytoplasm (Figures 5C and S5G: a, asterisks). Interestingly, in non-mitotic NIH-3T3 cells we detected PTTG1 in the primary cilium, a microtubule-based organelle, essential for SHH signaling. PTTG1 localized to a subset of primary cilia (Figures 5D, arrowheads and S5G: b). We found PTTG1 in the periciliary region (Figure $5 \mathrm{E}$ ) as well as along the shaft of the primary cilium (Figures 5F, 5G and S5G: $b-c)$. Within the shaft PTTG1 protein localization was heterogenous, sometimes covering only the proximal region of the cilium (Figures 5F and S5G: b) and in other cases the entire ciliary shaft (Figures 5G and S5G: $c$ ). The variable localization of PTTG1 amongst different cilia suggested that it is not a structurally required component of the primary cilium, but may be shuttled into the shaft in a regulated fashion to support ciliary function.

We further analyzed if PTTG1 is also localized to cilia of the developing brain using 
cephalic explants from wild type embryos. In neuroepithelial stem cells from both mouse strains we detected PTTG1 localization to a subset of primary cilia (Figure 6A, arrowheads), in the periciliary region (Figure 6B: a, d) and in the shaft of the primary cilium labeled for ARL13b (Figures 6B: b, c, e, f and S6B: a', a"-b', b").

Ultrastructural analysis revealed that PTTG1 was localized to the microtubule-based ciliary axoneme (Figure $6 \mathrm{C}$, boxed and $\mathrm{S} 6 \mathrm{C}$ ) and to the periciliary region at the appendages (Figures 6C, arrowhead and S6C, arrowheads). PTTG1 localization at the base of and in the primary cilium is depicted in a graphical model (Figure 6E).

Taken together our results identified PTTG1 as a novel microtubule-associated component of the primary cilium that can modulate efficiency of the canonical $\mathrm{SHH}$ signaling pathway.

\section{Cilia number and length differ between neuroepithelial stem cells from C57BL/6N and FVB/N wild type strains.}

Analyzing neuroepithelial stem cells from C57BL/6N and FVB/N cephalic explants revealed differences in cilia number per area (Figure S6B: a - b) and in cilia length between the strains (Figure 6D). Calculating the number of cilia in the anterior neural tube on the apical surface of the explants, we identified $645 \mathrm{cilia} / \mathrm{mm}^{2}$ surface area in FVB/N compared to $425 \mathrm{cilia} / \mathrm{mm}^{2}$ surface area in C57BL/6N explants (Figure S6B: a - b). We also used super resolution gated STED microscopy to image anterior neural tube explants from E9.5 C57BL/6N and FVB/N mice labeled for the regulatory GTPase $A R L 13 b$, which is localized to the membrane of the entire ciliary shaft. Strikingly, neuroepithelial stem cells of the rescue strain FVB/N had significantly shorter primary cilia compared to the HPE susceptible strain (Figure 6D). 

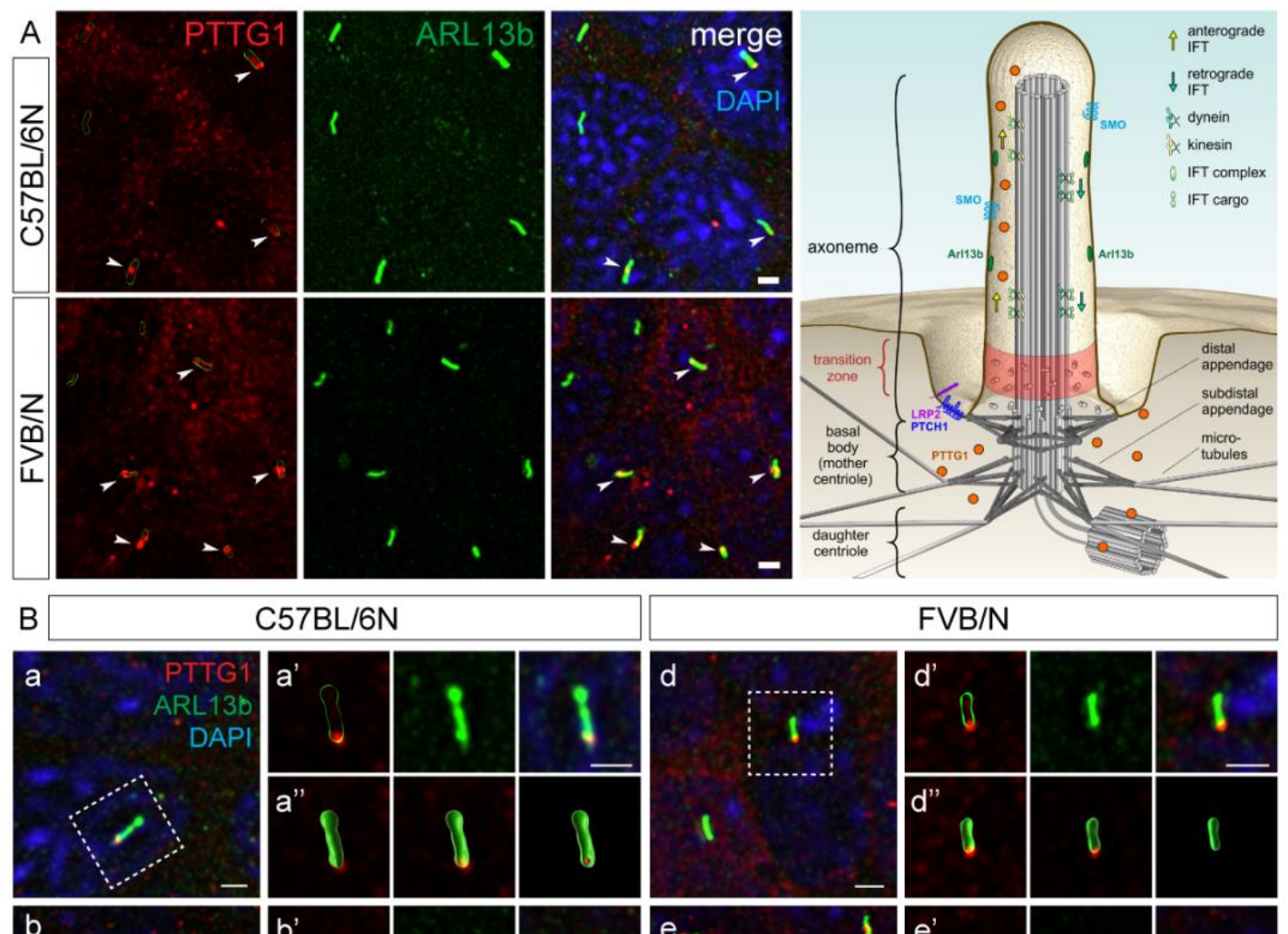

\section{FVB/N}
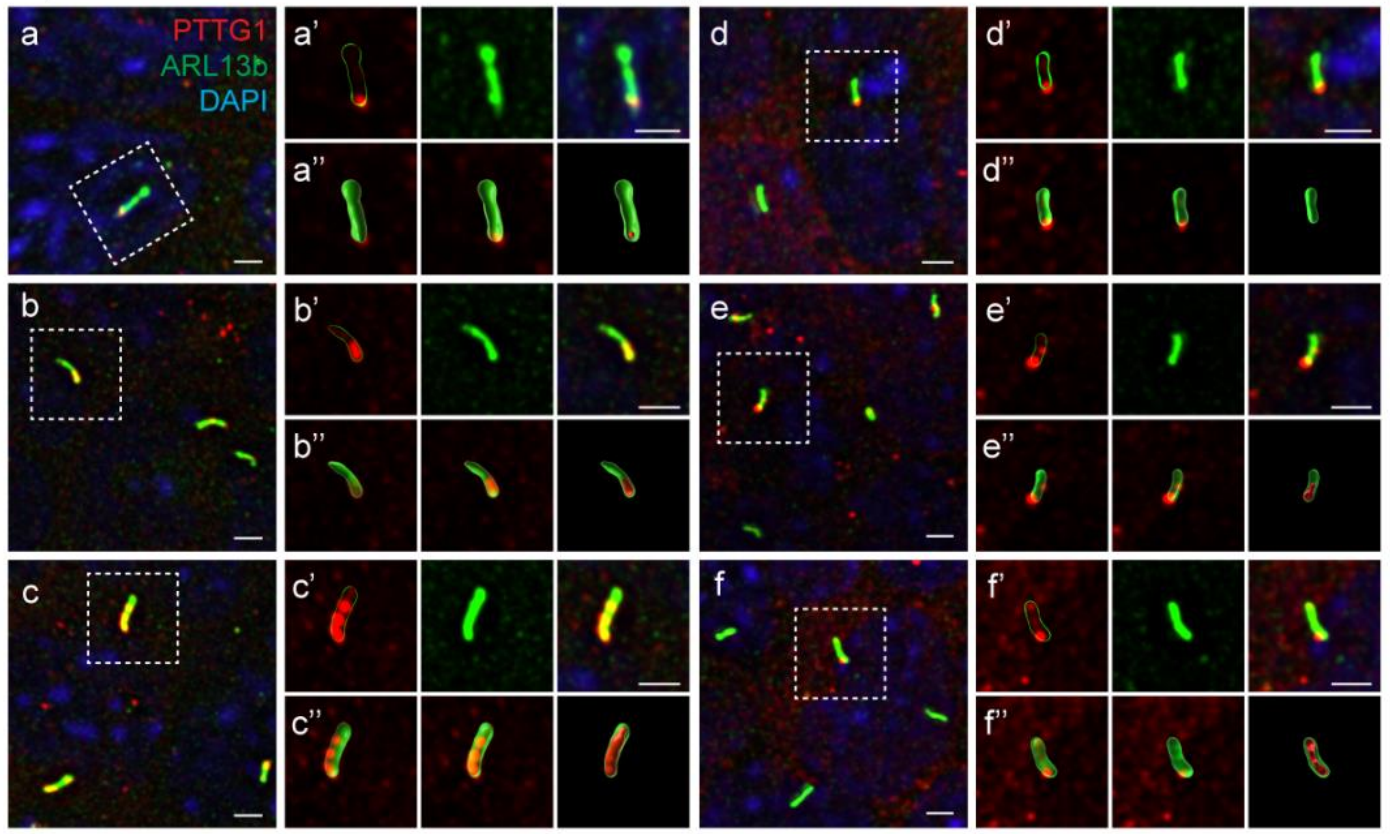

C
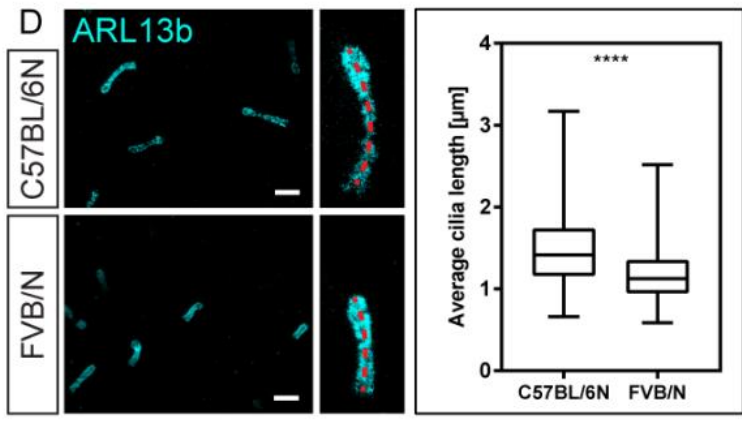

Figure 6: PTTG1 is a novel ciliary component in neuroepithelial stem cells

(A) Confocal microscopy on the E9.5 C57BL/6N $(n=6)$ and FVB/N $(n=6)$ cephalic explants shows PTTG1 in a subset of ARL13b positive primary cilia (arrowheads). Scale bars: $1 \mu \mathrm{m}$. 
(B) High resolution confocal 3D imaging: PTTG1 is present in the periciliary region (a and d) and in the ciliary shaft (b, $\mathbf{c}$ and $\mathbf{e}, \mathbf{f})$. Top panel insets (a' - f') show magnified cilia (dashed squares) with single signals for PTTG1 (with outlining the cilium), ARL13b and merge, respectively. Bottom panel insets (a" - f') show the same cilia as follows: reconstruction of ARL13b with non-reconstructed PTTG1 signal, optical section through this and reconstruction of both, ARL13b and PTTG1. Scale bars: $1 \mu \mathrm{m}$.

(C) Immunogold labeling of PTTG1 in the forebrain neuroepithelium of C57BL/6N embryos at E9.5 showed clear localization of PTTG1 at the microtubule-based axoneme in the shaft of the primary cilium. PTTG1 was also localized to the daughter centriole (arrow) suggesting a role in microtubule stabilization and assembly of primary cilia. PTTG1 was also detected in the periciliary region (arrowhead) and few signals were detected in the cytoplasm (asterisk). Scale bars: $100 \mathrm{~nm}$.

(D) Differences in primary cilia length comparing C57BL/6N and FVB/N neuroepithelial stem cells. Primary cilia labeled for ARL13b were imaged in the anterior region of E9.5 cephalic explants using STED microscopy. Primary cilia of $L r p 2^{+/+} F V B / N$ embryos $(n=7)$ were $20 \%$ shorter $(1.19 \mu \mathrm{m} \pm 0.011 \mathrm{SEM} ; \mathrm{n}=1068)$ compared to cilia from $\mathrm{Lrp2}^{+/+}$ C57BL/6N $(n=8)$ samples $(1.49 \mu \mathrm{m} \pm 0.014$ SEM; $n=908)$. Cilia length was measured as indicated by red dashed lines. The bar graph represents the mean cilia length with whiskers indicating minimal and maximal values and unpaired $t$-test for statistical analysis; ${ }^{* \star \star *} p<$ 0.00001 . Scale bars: $1 \mu \mathrm{m}$.

(E) Schematic of the primary cilium with components, relevant to the present work. PTTG1 is a novel ciliary component localized to the periciliary region and the ciliary shaft.

See also Figure S6. 


\section{DISCUSSION}

Lrp2 $2^{-/}$mutant mice studied on a C57BL/6N, a mixed C57BL/6N; 129/SvEMS-Ter, CD1, and a $\mathrm{C} 3 \mathrm{H} / \mathrm{HeNcrl}$ background show fully penetrant forebrain defects and perinatal lethality (Sabatino et al., 2017; Spoelgen et al., 2005; Willnow et al., 1996). We and others previously found that a $L r p 2^{267 / 267}$ ENU mutant line on a predominantly FVB/N background survived to adulthood (Gajera et al., 2010; Zarbalis et al., 2004; Zywitza et al., 2018). These studies prompted us to perform a more rigorous study using a pure congenic $L r p 2^{-/} \mathrm{FVB} / \mathrm{N}$ line to analyze the molecular mechanisms underlying these dramatic rescue effects.

Here we demonstrate that the FVB/N background rescued HPE and underlying SHH signaling defects, as well as the previously described heart outflow tract phenotype of Lrp2 $^{-1-}$ C57BL/6N mice (Baardman et al., 2016; Li et al., 2015). Thus, genetic background strongly modifies the severity of congenital forebrain and heart defects in LRP2 deficient mice. To our knowledge, this is the only case where a HPE related gene mutation leads to a fully penetrant phenotype on one background compared to $100 \%$ rescue on a second inbred strain. Mice carrying mutations in HPE genes and showing classic HPE features can display a lower penetrance or a milder phenotype on a different background strain, but these were not all or none effects. CDON (cell adhesion molecule-related/down-regulated by oncogenes) is a positive regulator of the SHH pathway (Hong and Krauss, 2018; Hong et al., 2020; Zhang et al., 2006); Cdon/- mice on a C57BL/6 background show HPE but only have microforms of HPE on a $129 S 6$ strain. Background modifier studies in early embryonic development where the FVB/N strain was used, are rare and could yet shed important light on the etiology of neural tube defects as reviewed in (Leduc et al., 2017). Exencephaly in Cecr2 mutant mice shows strain specific differences in penetrance comparing BALB/cCrl and FVB/N, with the latter being a rescue background. Whole genome linkage analysis revealed chromosome 19 as a modifier locus. In subsequent expression profiling of 
chromosome 19 by microarray analysis, the authors identified DEGs, including Arhgap19, which is expressed at lower levels in BALB/cCrl. A functional link between Arhgap19 expression levels and the phenotype penetrance was not investigated (Banting et al., 2005; Davidson et al., 2007; Kooistra et al., 2011).

In our study we combined transcriptome and functional analyses to identify disease relevant modifier genes. Our RNA sequencing approach identified Tuba1c and Ulk4 as the top down- and up-regulated DEGs, respectively, (Figure 4E and 4J). Functional data on Tuba1c regarding ciliogenesis or SHH pathway regulation are very limited. In the Reactome Pathway Knowledgebase Tuba1c is linked to the hedgehog $(\mathrm{HH})$ "off" state suggesting that this tubulin plays a role as a negative regulator in the $\mathrm{SHH}$ pathway (Fabregat et al., 2018; Rothfels, 2014).

Ulk4 is postulated to play an essential role in normal brain development and has been genetically linked to increased susceptibility to developing schizophrenia in humans (Lang et al., 2014). Mice with Ulk4 gene defects show, amongst other phenotypes, hydrocephaly, dilated brain ventricles and ependymal motile ciliary defects (Liu et al., 2016); but ULK4 function has not been associated with the SHH pathway before. It is hypothesized that ULK4 regulates neuronal function by acetylation of $\alpha$-tubulin, an important post-translational modification of microtubules (Lang et al., 2014, 2016). This is an interesting aspect since thereby both top hit DEGs, Ulk4 and Tuba1c, are associated with microtubule nucleation.

Here, we demonstrate a potential new function for ULK4 as it can enhance canonical SHH signaling capacity in a heterologous system (Figure 5A). Further, we suggest that ULK4 is a novel factor that might regulate the penetrance of $\mathrm{SHH}$ related congenital disorders.

In an unbiased approach we asked whether the top regulated modifier candidate gene Pttg1 could potentially also affect SHH activity. PTTG1 is a known substrate for the anaphase promoting complex (APC) and associates with separin until activation of the APC (Hagting et al., 2002; Mei et al., 2001; Thornton and Toczyski, 2003; Yanagida, 
2000). Various functions for PTTG1 have been described including control of mitosis, DNA repair, transcriptional activity, and cell migration (Genkai et al., 2006; Havens and Walter, 2011; Hellmuth et al., 2020; Holt et al., 2008; Xiang et al., 2017; Yan et al., 2015; Zheng et al., 2015). The protein has tumorigenic activity and the gene is amplified in various human tumors of the breast, uterus, lung and thyroid ${ }^{74-78}$. High expression of Pttg1 has been also correlated with aggressive forms of brain tumors, including medulloblastoma and glioblastoma (Salehi et al., 2013; Yan et al., 2015). During embryonic development Pttg1 expression has been reported in the human and murine brain (Boelaert et al., 2003; Karsten et al., 2003; Tarabykin et al., 2000). A recent study demonstrated a role of PTTG1 in microtubule nucleation (Moreno-Mateos et al., 2011). However, PTTG1 has so far not been associated with microtubule-based axoneme function of the primary cilium or with canonical $\mathrm{SHH}$ pathway modulation in the cilium. Here we show that Pttg1 overexpression can indeed modulate $\mathrm{SHH}$ responsiveness in transfected cells (Figure 5A). Therefore, we conclude that PTTG1 is a new $\mathrm{SHH}$ pathway component and can promote canonical $\mathrm{SHH}$ signaling efficiency.

We also discovered a novel and seemingly dynamic localization of PTTG1 to the periciliary region and to microtubule-based axoneme of the cilium in quiescent/interphase NIH-3T3 and in neuroepithelial stem cells of the developing brain (Figures 5, S5, 6 and S6). The ciliary localization supports the hypothesis that PTTG1 modulates the SHH pathway via cilia-associated mechanisms. So far, only a relatively small number of regulatory proteins have been reported to be involved in both cytokinesis and ciliogenesis (Gromley et al., 2005; Kim et al., 2005; Pan et al., 2007; Park et al., 2008; Shah et al., 2008; Smith et al., 2011; Spektor et al., 2007; Vertii et al., 2015; Zuo et al., 2009). The anaphase promoting complex (APC), important for cytokinesis, is found to be also localized to the basal body of the primary cilium during interphase where its activity regulates disassembly of the primary cilium (Wang et al., 2014). 
Our data provide evidence that PTTG1, besides its function as a cell cycle regulator associated with the centrosome, also plays a novel role in ciliogenesis and ciliary function, e.g. SHH signaling in interphase/quiescent cells. We propose that higher expression levels of PTTG1 predispose neuroepithelial stem cells in FVB/N mice to more efficient $\mathrm{SHH}$ signaling by enhancing microtubule repolymerization of the ciliary axoneme after cell division (Moreno-Mateos et al., 2011). Knockdown of PTTG1 has been shown to attenuate microtubule repolymerization after nocodazole treatment, which leads to microtubule depolymerization (Moreno-Mateos et al., 2011). Efficient microtubule repolymerization after cell division is crucial for assembly of the primary cilium and therefore $\mathrm{SHH}$ signaling (He et al., 2017). In our study, enhanced ciliogenesis is also supported by in average higher number of cilia per area in the FVB/N neuroepithelium compared to C57BL/6N.

Interestingly, all three top regulated modifier candidate genes, Tuba1c, Ulk4, and Pttg1, identified in our screen, are linked to microtubule function, suggesting that the expression profile of these genes in a FVB/N background provides an advantageous environment for the microtubule based ciliary function and $\mathrm{SHH}$ signaling, and ultimately a protective effect against developmental defects (Figure 7). These factors are most likely not necessary for establishing $\mathrm{SHH}$ signaling but have positive effects on the overall efficiency of the pathway. Combinatorial expression profiles of these candidate modifier genes, and likely other still uncharacterized modifier candidates, ensure sufficient SHH activity in the absence of LRP2 on a FVB/N background.

Differences in ciliary factors between strains could be also reflected in different morphology of primary cilia. Indeed, our super resolution imaging revealed striking differences in the morphology of primary cilia between C57BL/6N and FVB/N neuroepithelial stem cells (Figures 6 and S6), suggesting that the genome of wild type mice from different mouse strains has a substantial influence on the shape of primary cilia. This could have functional consequences and relevant clinical implications considering the complex signaling function of the primary cilium (Gigante and Caspary; 
Goetz and Anderson, 2010; Nachury and Mick, 2019; Singla and Reiter, 2006). Little is known about the regulation of cilia length and the implication of variable cilia length in health and disease including ciliopathies (Fliegauf et al., 2007; Gerdes et al., 2009). Interactions of ciliary components, e.g. prominin with ARL13b, have been shown to play a role in maintenance of length of the primary cilia throughout the animal kingdom (Jászai et al., 2020). Studies in mammalian cells suggest that increased soluble tubulin production leads to longer cilia and cytosolic tubulin stabilization results in shorter cilia (Sharma et al., 2011; Wang and Dynlacht, 2018; Wang et al., 2013). Wang et al. showed that the complex formed by cell cycle regulator APC and its co-activator Cdc20 at the basal body maintains the optimal ciliary length and is crucial to shorten the cilia when cells exit from quiescent stage (Wang et al., 2014). Since PTTG1 is a substrate for APC (Mei et al., 2001; Yanagida, 2000), and also found at the base of the cilium in our study, this conceptually links the function of PTTG1 to cilia length regulation.

We conclude that the $\mathrm{FVB} / \mathrm{N}$ inbred mouse strain has advantageous ciliary composition and morphology and identify plausible molecular candidates that render the strain more resilient to disturbances of the SHH pathway (Figure 7). Our study highlights the importance of mouse strain dependent penetrance of phenotypes. The expression profiles that differ profoundly between different wild type strains during early developmental stages provide a unique resource, that to our knowledge has not been available before. Considering the huge effects of the FVB/N background this resource is particularly valuable to understand genetic robustness in brain and heart development. Furthermore, we show that genetics in the mouse combined with functional cell biological studies can define novel molecular mechanisms that potentially underly variability in the penetrance of human cilia-associated neurodevelopmental disorders. Identification of disease relevant modifier genes in the mouse can provide important insights into the etiology and prevention of human congenital disorders. 


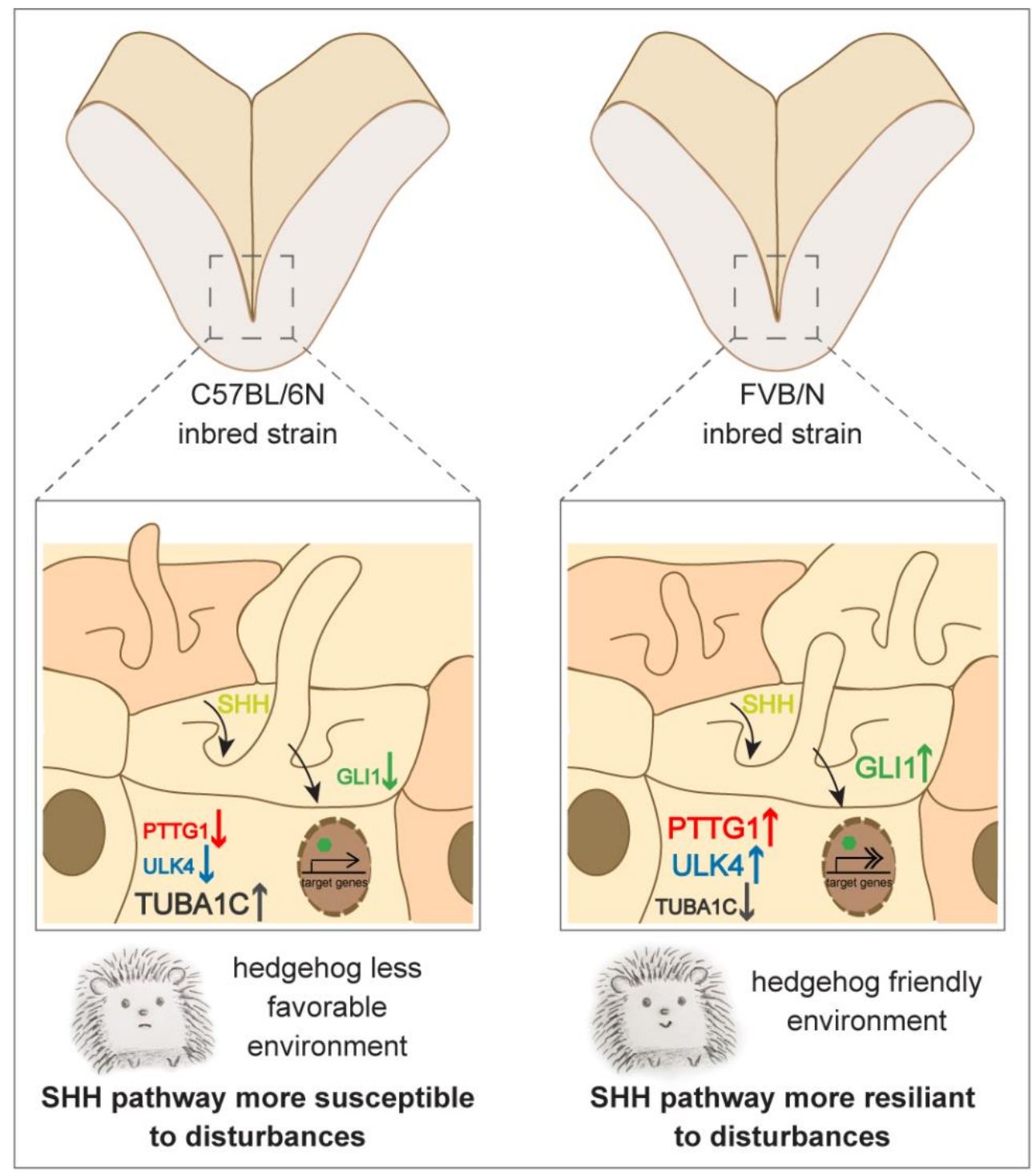

Figure 7: Proposed model: Sufficient SHH signaling is crucial for proper specification of the developing ventral neural tube and therefore essential for subsequent separation of the cortical hemispheres. Strain specific expression of candidate modifier genes leave the neuroepithelial stem cells of C57BL/6N embryos more susceptible to disturbances of the $\mathrm{SHH}$ pathway and therefore to congenital brain disorders such as HPE. FVB/N neuroepithelial stem cells with higher Pttg1 and Ulk4 and lower Tuba1c expression show enhanced SHH signaling and seem to be more resilient to disturbances of the SHH pathway. TUBA1c, ULK4, and PTTG1 are linked to microtubule function and PTTG1 is localized to the ciliary axoneme. The levels of the modifiers could enhance microtubule repolymerization of the ciliary axoneme after cell division and thereby facilitate $\mathrm{SHH}$ signaling. Further, in average shorter primary cilia could contribute to an overall more hedgehog friendly environment in FVB/N compared to C57BL/6N neural tissue. 


\section{ACKNOWLEDGEMENTS:}

We are grateful to Manfred Ströhmann for excellent work in the mouse husbandry. We thank Anke Scheer for excellent technical assistance. Wei Chen and Mirjam Feldkamp from the MDC genomics platform performed RNA deep sequencing. Alexandra KlausBergmann provided helpful discussions on the study. Petra Schrade helped with scanning electron microscopy. Christina Schiel performed immunogold electron transmission microscopy. We thank Thomas Willnow for acquisition of financial support for the project. We would like to thank Gary Lewin for helpful discussions, intellectual input and critical reading of the manuscript. We are grateful to David Mick for critically reading the manuscript and intellectual input.

\section{FUNDING SOURCES:}

Funding was provided by the German Research Foundation (DFG): SFB665, SFB958, GRK2318/1

\section{AUTHOR CONTRIBUTION}

N.M. conceived and performed experiments and analyzed data. I.K. conceived and performed experiments, analyzed data, and wrote parts of the manuscript. F.W. performed DESeq2 analysis and analyzed data, J.G. and A.L. performed experiments and analyzed data. H.G. and M.L. helped with STED microscopy and data analysis. M.R. and A.S. helped with confocal microscopy data analysis. B.P. carried out immunogold electron microscopy analysis. N.H. provided intellectual input, and edited the manuscript. A.H. conceptualized and supervised the study, secured funding and wrote the manuscript.

\section{DECLARATION OF INTERESTS}

The authors declare no competing interests. 


\section{METHODS}

KEY RESOURCES TABLE

\begin{tabular}{|c|c|c|}
\hline REAGENTS & SOURCE & IDENTIFIER \\
\hline \multicolumn{3}{|l|}{ Antibodies } \\
\hline rabbit anti-SHH $(\mathrm{SHH}(\mathrm{H}-160)$ & Santa Cruz & Cat. \#sc9024 \\
\hline rat anti-SHH & R\&D & Cat. \#MAB4641 \\
\hline sheep anti-LRP2 & $\begin{array}{l}\text { Laboratory of } \\
\text { Renata Kozyraki }\end{array}$ & $\mathrm{N} / \mathrm{A}$ \\
\hline rabbit anti-LRP2 & Abcam & Cat. \#ab76969 \\
\hline rabbit anti-PTTG1 & Abcam & Cat. \#ab79546 \\
\hline goat anti-PTTG1 & $\begin{array}{l}\text { LifeSpan } \\
\text { Biosciences }\end{array}$ & Cat. \#LS-B5119-50 \\
\hline rabbit anti- ARL13b & ProteinTech & Cat. \#17711-1-AP \\
\hline mouse anti- ARL13b & $\begin{array}{l}\text { UC Davis/NIH } \\
\text { NeuroMab }\end{array}$ & Cat. \#75-287 \\
\hline mouse anti- acetylated Tubulin & Sigma Aldrich & Cat. \#T7451 \\
\hline mouse anti- gamma Tubulin & Sigma Aldrich & Cat. \#T6557 \\
\hline mouse anti- alpha Tubulin & Merck Millipore & Cat. \#CP06 \\
\hline Donkey anti-sheep Alexa Fluor 488 & Abcam & Cat. \#ab150177 \\
\hline Donkey anti-rabbit Alexa Fluor 488 & Abcam & Cat. \#ab150073 \\
\hline Donkey anti-mouse Alexa Fluor 488 & Abcam & Cat. \#ab150109 \\
\hline Donkey anti-rabbit Alexa Fluor 555 & Abcam & Cat. \#ab150074 \\
\hline Donkey anti-mouse Alexa Fluor 555 & Abcam & Cat. \#ab150106 \\
\hline Donkey anti-mouse Alexa Fluor Plus 594 & Invitrogen & Cat. \#A32744 \\
\hline Donkey anti-mouse Alexa Fluor 647 & Abcam & Cat. \#ab150107 \\
\hline Donkey anti-goat Alexa Fluor 647 & Abcam & Cat. \#ab150131 \\
\hline Goat anti-rabbit - Gold 12nm & Dianova & Cat. \#111-205-144 \\
\hline DAPI & Invitrogen & Cat. \#62248 \\
\hline \multicolumn{3}{|c|}{ Chemicals, Peptides, and Recombinant Proteins } \\
\hline Cresol Red sodium salt & Sigma Aldrich & Cat. \#114480 \\
\hline Hematoxylin & Carl Roth & Cat. \#T865 \\
\hline Eosin & Carl Roth & Cat. \#X883.2 \\
\hline Roti Histokit & Carl Roth & Cat. \#6638.1 \\
\hline RotiHistol & Carl Roth & Cat. \#6640.1 \\
\hline
\end{tabular}




\begin{tabular}{|c|c|c|}
\hline Poly vinyl alcohol & Sigma Aldrich & Cat. \#P8136 \\
\hline Tissue-Tek ${ }^{\circledR}$ O.C.T. ${ }^{\text {TM }}$ Compound & Sakura Finetek & Cat. \#sa-4583 \\
\hline Paraffin & Carl Roth & Cat. \#6642.1 \\
\hline Paraformaldehyde & Sigma Aldrich & Cat. \#16005 \\
\hline Donkey serum & Biowest & Cat. \#S2170-500 \\
\hline Dako fluorescence mounting medium & Agilent & Cat. \#S302380-2 \\
\hline DMEM, high glucose, GlutaMAX ${ }^{\text {тM }}$ Supplement & Invitrogen & Cat. \#31966047 \\
\hline Fetal Bovine Serum & PAN-Biotech GmbH & Cat. \#P40-37500 \\
\hline Penicillin-Streptomycin & Invitrogen & Cat. \#15140122 \\
\hline Lipofectamine3000 & Invitrogen & Cat. \#L3000015 \\
\hline D-Luciferin, Potassium Salt & ZellBio & Cat. \#LUCK-100 \\
\hline Coelenterazine & ZellBio & Cat. \#CZ2.5 \\
\hline KAAD-cyclopamine & Calbiochem & Cat. \#239804 \\
\hline Tris- $\mathrm{HCl}$ & Carl Roth & Cat. \#9090.5 \\
\hline SDS & Serva & Cat. \#20765.03 \\
\hline Glycerol & Carl Roth & Cat. \#3783.1 \\
\hline bromophenol blue & Merck & Cat. \#11746.0005 \\
\hline$\beta$-mercaptoethanol & Carl Roth & Cat. \#4227.3 \\
\hline $12 \%$ Tris-Glycine Gel & Invitrogen & Cat. \#XP0012C \\
\hline Nitrocellulose membrane $0.2 \mu \mathrm{m}$ & Amersham Protran & Cat. \#10600006 \\
\hline SuperSignal West Dura & Life Technologies & Cat. \#34075 \\
\hline Slow Fade Diamond Antifade Mountant & Invitrogen & Cat. \#S36963 \\
\hline ProLong Gold Antifade Mountant & Invitrogen & Cat. \#P36982 \\
\hline \multicolumn{3}{|l|}{ Critical Commercial Assays } \\
\hline DIG RNA labeling kit & Roche & $\begin{array}{l}\text { Cat. } \\
\# 11277073910\end{array}$ \\
\hline Batson's \#17 Anatomical Corrosion Kit & Polyscience & Cat. \#07349 \\
\hline RNeasy Plus Micro kit & Qiagen & Cat. \#74034 \\
\hline RNA 6000 Nano kit & Agilent & Cat. \#5067-1511 \\
\hline TruSeq Stranded mRNA protocol & Illumina & Cat. \#20020594 \\
\hline High capacity RNA-to-cDNA kit & Life technologies & Cat. \#4387406 \\
\hline TaqMan Universal PCR Master Mix & Life technologies & Cat. \# 4304437 \\
\hline Gli1 Taqman probe & Life technologies & $\begin{array}{l}\text { Cat. } \\
\text { \#Mm00494654_m } \\
1\end{array}$ \\
\hline
\end{tabular}




\begin{tabular}{|c|c|c|}
\hline Gapdh Taqman probe & Life technologies & $\begin{array}{l}\text { Cat. } \\
\text { \#Mm99999915_g1 }\end{array}$ \\
\hline \multicolumn{3}{|l|}{ Experimental Models: Cell Lines } \\
\hline murine NIH-3T3 & AG Willnow & (Christ et al., 2012) \\
\hline human HEK293T & AG Willnow & (Christ et al., 2012) \\
\hline human SHHN-293 & laboratory of M. Kato & (Christ et al., 2012) \\
\hline \multicolumn{3}{|l|}{ Experimental Models: Mouse } \\
\hline $\mathrm{Lrp2}^{+/-} \mathrm{C} 57 \mathrm{BL} 6 / \mathrm{NCrl}$ & $\begin{array}{l}\text { (Spoelgen et al., } \\
2005)\end{array}$ & $\mathrm{N} / \mathrm{A}$ \\
\hline Lrp2 $^{+/-} \mathrm{FVB} / \mathrm{NCrl}$ & & $\mathrm{N} / \mathrm{A}$ \\
\hline \multicolumn{3}{|l|}{ Oligonucleotides } \\
\hline Lrp2 PCR primer, targeted allele forward & Eurofins & $\begin{array}{lll}\text { GAT } & \text { TGG } \\
\text { GAC } & \text { AAT } \\
\text { AGG } & \text { CAT GC }\end{array}$ \\
\hline $\begin{array}{l}\text { Lrp2 PCR primer, wild type and targeted allele } \\
\text { reverse }\end{array}$ & Eurofins & $\begin{array}{lll}\text { CAT } & \text { ATC } & \text { TTG } \\
\text { GAA } & \text { ATA } & \text { AAG } \\
\text { CGA C } & \end{array}$ \\
\hline Lrp2 PCR primer, wild type allele forward & Eurofins & $\begin{array}{lll}\text { GAC } & \text { CAT } & \text { TTG } \\
\text { GCC } & \text { AGC } & \text { CAA } \\
\text { GG } & & \end{array}$ \\
\hline
\end{tabular}

\section{Recombinant DNA:}

\begin{tabular}{|l|l|l|}
\hline Firefly luciferase reporter (8 x 3'Gli-BSס51Lucll) & (Sasaki et al., 1997) & N/A \\
\hline Renilla luciferase reporter (pRL-TK) & Promega & Cat. \#E2241 \\
\hline cDNA Pttg1 & Origene & Cat. \#MR202008 \\
\hline cDNA Ulk4 & Origene & Cat. \#MR217918 \\
\hline B9d1 RIKEN cDNA clone & Source Bioscience & Cat. \# 2810407E13 \\
\hline Tuba1c RIKEN cDNA clone & Source Bioscience & Cat. \#2810407E13 \\
\hline Nkx2.1 riboprobe & Carmen Birchmeier & $\begin{array}{l}\text { (NM_009385.2, } \\
\text { bps 2032- 2813) }\end{array}$ \\
\hline Shh riboprobe & Andrew P. McMahon & $\begin{array}{l}\text { (NM_009170.3, } \\
\text { bps 455-1097) }\end{array}$ \\
\hline Software and Algorithms: & &
\end{tabular}

\section{Software and Algorithms:}

Centro XS $^{3}$ LB 960 Microplate Luminometer, Mikrotek

Berthold Technologies, MikroWin 20004.41 Laborsysteme

software $\quad \mathrm{GmbH}$ 


\begin{tabular}{|c|c|c|}
\hline $\begin{array}{l}\text { BioRad CFX384 Real Time System and CFX } \\
\text { Manager } 3.1\end{array}$ & BioRad & \\
\hline DESeq2 (v1.12.4) & (Love et al., 2014) & $\begin{array}{l}\text { http://bioconductor. } \\
\text { org/packages/3.6/b } \\
\text { ioc/html/DESeq2.h } \\
\text { tml }\end{array}$ \\
\hline $\begin{array}{l}\text { TopHat (v2.0.12) } \\
\text { and Bowtie 2.0.6.0 }\end{array}$ & $\begin{array}{l}\text { (Trapnell et al., } \\
2009)\end{array}$ & $\begin{array}{l}\text { https://ccb.jhu.edu/ } \\
\text { software/tophat/do } \\
\text { wnloads/tophat- } \\
\text { 2.0.12.tar.gz }\end{array}$ \\
\hline $\begin{array}{l}\text { SR TruSeq Cluster Kit V3 } \\
\text { Illumina High Seq2000 HCS 2.2.38 }\end{array}$ & & $\begin{array}{l}\text { https://support.illu } \\
\text { mina.com/content/ } \\
\text { dam/illumina- } \\
\text { support/documents } \\
\text { /documentation/sy } \\
\text { stem_documentati } \\
\text { on/translations/his } \\
\text { eq-2000-system- } \\
\text { guide-15011190- } \\
\text { deu.pdf }\end{array}$ \\
\hline GraphPad Prism 7 & $\begin{array}{l}\text { GraphPad Software, } \\
\text { Inc }\end{array}$ & $\begin{array}{l}\text { https://www.graph } \\
\text { pad.com/scientific- } \\
\text { software/prism/ }\end{array}$ \\
\hline ImageJ & & $\begin{array}{l}\text { http://imagej.nih.go } \\
\text { v/ij/ }\end{array}$ \\
\hline Leica LAS V4.9 imaging software & Leica & \\
\hline Leica LAS X 3.3.3. imaging software & Leica & \\
\hline Huygens Professional 19.10 & $\begin{array}{l}\text { Scientific Volume } \\
\text { Imaging }\end{array}$ & $\begin{array}{l}\text { https://svi.nl/Home } \\
\text { Page }\end{array}$ \\
\hline IMARIS 9.3 and 9.5 & Bitplane & $\begin{array}{l}\text { https://imaris.oxins } \\
\text { t.com/packages }\end{array}$ \\
\hline Adobe Illustrator 2019 & Adobe & $\begin{array}{l}\text { https://www.adobe. } \\
\text { com/products/illust } \\
\text { rator.html\# }\end{array}$ \\
\hline
\end{tabular}




\begin{tabular}{|l|l|l|}
\hline Adobe Photoshop CC 2019 & Adobe & $\begin{array}{l}\text { https://www.adobe. } \\
\text { com/products/phot } \\
\text { oshop.html }\end{array}$ \\
\hline iTEM software & EMSIS GmbH & $\begin{array}{l}\text { https://www.itemso } \\
\text { ft.com/ }\end{array}$ \\
\hline Other & & \\
\hline Nunc Lab-Tek II Chamber slides & Sigma Aldrich & Cat. \#C6807 \\
\hline Mini Gel Tank & Invitrogen & Cat. \#A25977 \\
\hline Millicell Hanging Cell Culture Insert & Merck Millipore & Cat. \#MCEP06H48 \\
\hline Secure-Seal Spacer & Invitrogen & Cat. \#S24737 \\
\hline sub-resolution TetraSpeck ${ }^{\text {TM }}$ Microspheres & Invitrogen & Cat. \#T7280 \\
\hline
\end{tabular}

RESOURCE AVAILABILITY

\section{Lead Contact}

Further information and requests for resources and reagents should be directed to and will be fulfilled by the Lead Contact, Annette Hammes (hammes@mdcberlin.de).

\section{Materials Availability}

This study did not generate new unique reagents. Lrp2 ${ }^{+-}$mouse line on FVB/N background will be available upon request.

\section{Data and Code Availability}

The RNA sequencing datasets generated in this study have been made available as Supplementary Table 2 (excel file). 


\section{EXPERIMENTAL MODEL AND SUBJECT DETAILS}

\section{Animals}

Experiments involving animals were performed according to institutional guidelines following approval by local authorities (X9005/12). Mice were housed in a 12 hours light-dark cycle with ad libitum food and water. The generation of mice with targeted disruption of the Lrp2 gene has been described before (Willnow et al., 1996). The Lrp2 mutant mouse line was crossed onto a pure C57BL/6NCrl background in our laboratory (Spoelgen et al., 2005) (herein referred to as Lrp2 mutant C57BL/6N. For this study the $\mathrm{Lrp2}^{+/-} \mathrm{C} 57 \mathrm{BL} / 6 \mathrm{~N}$ line was backcrossed for more than 12 generations to obtain a congenic mouse line on a FVB/NCrl background (herein referred to as Lrp2 mutant on FVB/N background).

We used mice $>8$ weeks of age for timed matings for our experiments. Analyses of the congenital defects were carried out in $\mathrm{Lrp}^{-/-}$and in somite matched $\mathrm{Lrp2}^{+/+}$and/or $\mathrm{Lrp2}^{+/-}$control littermates on a C57BL/6NCrl and FVB/NCrl background. For the generation of the $\mathrm{F} 1$ hybrid Lrp2 mutant embryos, Lrp2 ${ }^{+/} \mathrm{FVB} / \mathrm{NCrl}$ females were crossed with $\mathrm{Lrp2}^{+/-} \mathrm{C} 57 \mathrm{BL} / 6 \mathrm{NCrl}$ males. PCR genotyping was performed from yolk sac genomic DNA for E8.5 embryos and from tail biopsies for older embryos.

\section{METHOD DETAILS}

\section{Histology}

Standard NISSL and Hematoxylin \& Eosin staining were performed on paraffin sections. Embryos were fixed in $4 \%$ paraformaldehyde in PBS at $4^{\circ} \mathrm{C}$, overnight, embedded in paraffin and cut with $10 \mu \mathrm{m}$ thickness. NISSL staining was performed in 0.1\% Cresol Red solution (Sigma Aldrich, Cat. \#114480) for 3-10 minutes. Hematoxylin \& Eosin staining was performed according to manufacturer's instructions 
with incubation times of 5 min in Hematoxylin (Carl Roth, Cat. \#T865) and 3 min in Eosin (Carl Roth, Cat. \#X883.2). Sections were dehydrated and mounted in Roti Histokit (Carl Roth, Cat. \#6638.1). Staining was visualized using the Leica DM5000B microscope using the LAS-X 3.3.3. Software.

\section{In situ Hybridization}

Whole mount in situ hybridization (WISH) was carried out as described previously (Hammes et al., 2001). In situ hybridization (ISH) on sections was performed as described in (Jensen and Wallace, 1997), except that the signal was enhanced by performing the color reaction in the presence of $10 \%$ poly vinyl alcohol (Sigma Aldrich, Cat. \#P8136). Probe synthesis was conducted with the components of the DIG RNA labeling kit (Roche, Cat. \#11277073910). Plasmids for generating in situ probes were generated from the following mRNA sequences: Nkx2.1 (NM_009385.3, bps 2032 2813) kindly provided by Carmen Birchmeier, Shh (NM_009170.3, bps 455 - 1097) kindly provided by Andrew P. McMahon (University of Southern California).

\section{Injection of heart samples with Batson's pigment}

Polymeric dye injections were used to visualize the ascending aorta and the pulmonary artery in isolated E18.5 mouse hearts applying the Batson's \#17 Anatomical Corrosion Kit (Polyscience, Cat. \#07349). Batson's \#17 Blue pigment was added to Base Solution $\mathrm{A}$ in the amount of $2 \%$, mixed vigorously and divided into two equal parts. 24 $\mathrm{ml}$ of the Catalyst was added to the first $100 \mathrm{ml}$ of Base Solution A/pigment mix. 24 drops of Promoter $\mathrm{C}$ were carefully added to the second half of the Base Solution A/pigment and mixed slowly. Both solutions were mixed together and stirred. After both parts were mixed it took 30 to 45 minutes until the solution was fully polymerized and until then the experiment should be finished. The same procedure was repeated for Batson's \#17 Red pigment. Injections of the blue and red pigment solution into the right and left ventricle, respectively, were made with a disposable polyethylene syringe and 
23G needle under a stereomicroscope (Leica MZ 10F). Images of the heart were taken after the injection (Leica LAS V4.9 imaging software).

\section{RNA library generation and sequencing}

For the generation of an RNA library embryonic heads of E9.5 embryos were dissected, snap frozen and stored at $-80^{\circ} \mathrm{C}$ until RNA isolation. RNA was extracted using RNeasy Plus Micro kit (Qiagen, Cat. \#74034), checked on Bioanalyzer (Agilent RNA 6000 Nano kit, Cat. \#5067-1511), and samples with RIN>9 were used to prepare the cDNA library. Library preparation for mRNA sequencing was performed according to the Illumina SR TruSeq Stranded mRNA protocol (Cluster Kit V3, Illumina, Cat. \#20020594) on 23 embryonic head RNA samples from mice with 24 somites. Sequencing was performed on the Illumina HiSeq2000 system with HCS 2.2.38 software.

Sequencing reads were aligned to a SNP-infused (FVB/NCrl and C57BL/6NCrl SNPs, accordingly) mouse genome (Ensembl GRCm38.77) using TopHat v2.0.12 with Bowtie 2.0.6.0. The number of reads that mapped to a gene was counted using the HTseqcount v0.6.0 with default parameters. All expressed genes $(n=10,861)$ have been used for differential expression analysis using DESeq2 v1.12.4 (Love et al., 2014). We defined genes as differentially expressed meeting our significance threshold of FDR $\leq$ 0.05. Allele specific expression was determined by counting the reads matching the $\mathrm{C} 57 \mathrm{BL} / 6 \mathrm{~N}$ or $\mathrm{FVB} / \mathrm{N}$ genotype in the $\mathrm{F} 1$ mRNA-seq data.

\section{Differential gene expression analysis}

mRNA-seq quantifications were derived from exon-mapped, paired-end reads. Expression quantification was followed by read normalization, size factor estimation and differential expression analysis using DESeq2 v1.12.4 (Love et al., 2014).

For this analysis we included all genes that we consider to be expressed, defined as having at least 100 reads in 23 out of 26 samples $(n=10,861)$. We considered a gene 
differentially expressed when it met genome-wide significance thresholds of FDR $\leq$ 0.05. We performed three different comparisons: a) genotype comparison, where we assessed expression differences for each mouse strain separately (C57BL/6N, FVB/N or the F1 strain) comparing $L r p 2^{-/}$versus $L r p 2^{+/+}$samples; b) strain comparison, where the different mouse strains, C57BL/6N versus FVB/N and F1, were compared in Lrp2 ${ }^{+/+}$ and $L r p 2^{-/}$samples; and c) the interaction of genotype and strain effects, where the interplay of strain and LRP2-deficiency was assessed to understand strain differences that were observed between $L r p 2^{+/+}$versus $L r p 2^{-/}$samples.

\section{Real-Time Quantitative Reverse Transcription PCR (Real-Time qRT-PCR)} Total RNA from E9.5 embryos heads was isolated and cDNA was synthesized by High capacity RNA-to-cDNA kit (Life technologies, Cat. \#4387406). Quantitative PCR was performed using TaqMan Universal PCR Master Mix (Life technologies, Cat. \#4304437) with the BioRad CFX384 Real Time System used on a BioRad C1000 Touch Thermal Cycler. The expression of Gli1 (Taqman probe, Life Technologies, Cat. \#Mm00494654_m1) was normalized to Gapdh (Life Technologies, Cat. \#Mm99999915_g1). Transcript levels relative to Gapdh were calculated using the deltaCt method. Data were analyzed in GraphPad Prism 7 (GraphPad Software, Inc) using an unpaired t-test.

\section{Cell Culture}

The NIH-3T3 and HEK293T cell lines were obtained from T. Willnow (MDC) (Christ et al., 2012). SHHN-293 cells were originally kindly provided by M. Kato (Stanford School of Medicine). Cells were maintained in DMEM (Invitrogen, Cat. \#31966047) with 10\% Fetal Bovine Serum (FCS, PAN-Biotech GmbH, Cat. \#P40-37500) and 1\% PenicillinStreptomycin (Invitrogen, Cat. \#15140122). Cells were transfected with Lipofectamine $^{\text {TM }} 3000$ Transfection Reagent (Invitrogen, Cat. \#L3000015) according to the manufacturer's instructions. 
The following overexpression constructs were used: cDNA Pttg1 (Origene, Cat. \#MR202008), cDNA Ulk4 (Origene, Cat. \#MR217918), B9d1 RIKEN cDNA clone (Source Bioscience, Cat. \#0710008G05), Tuba1c RIKEN cDNA clone (Source Bioscience, Cat. \#2810407E13).

\section{Dual luciferase reporter assay}

NIH-3T3 cells, seeded in 24-well plates, were transiently co-transfected with the gene of interest or proper empty plasmid, respectively, GLI-dependent firefly luciferase reporter (8 x 3'Gli-BSס51Lucll) (Sasaki et al., 1997) and a constitutive Renilla luciferase reporter (pRL-TK; Promega, Cat. \#E2241) at a ratio 10:10:1 using Lipofectamine3000, according to manufacturer's protocol.

$24 \mathrm{~h}$ later the medium was replaced with conditioned medium from HEK293 cells stably secreting SHH-Np (SHHN-293 cells; kindly provided M. Kato, Stanford School of Medicine) or control medium from parental HEK293T cells, at a 1:10 dilution, in medium containing $0.5 \%$ FCS. For control studies KAAD-cyclopamine (Calbiochem, Cat. \#239804) at a $50 \mathrm{nM}$ concentration or solvent were added to the conditioned medium with SHH-Np.

After 48 hours of stimulation, activity was assayed with D-luciferin (ZellBio GmbH, Cat. \#LUCK-100) and results were normalized to the corresponding Renilla activity, assayed with coelenterazine (ZellBio $\mathrm{GmbH}$, Cat. \#CZ2.5). Software for Dual Luciferase reporter assay measurements: Centro XS XB $^{3}$ LB 960 Microplate Luminometer, Berthold Technologies, MikroWin 20004.41 software from Mikrotek Laborsysteme $\mathrm{GmbH}$. Experiments were performed in triplicates, referring to three different wells assayed the same day, in minimum of 3 independent experiments, and results are shown as mean and standard deviation. Statistical analyses were performed using Two-way ANOVA with Dunnett's multiple comparisons test. 


\section{Western Blot Analysis}

E8.5 whole embryos were lysed in SDS lysis buffer (60 mM Tris-HCl pH-6,8, 2\% SDS, $10 \%$ glycerol, $0.01 \%$ bromophenol blue, $1.25 \%$ beta-mercaptoethanol). Samples were heated to $95^{\circ} \mathrm{C}$ for $10 \mathrm{~min}$, centrifuged for $2 \mathrm{~min}$ at $13,200 \mathrm{rpm}$ and stored at $-20^{\circ} \mathrm{C}$ until used. Equal amounts of samples were subjected to a $12 \%$ Tris-Glycine Gel (Invitrogen, Cat. \#XP0012C) in a Mini Gel Tank (Invitrogen, Cat. \#A25977). The resolved proteins were transferred onto a nitrocellulose membrane (Amersham Protran $0.2 \mu \mathrm{m}$, Cat. \#10600006) using a wet electroblotting system (Bio-Rad Mini Protean II Cell) followed by immunoblotting. $5 \%$ non-fat dry milk in TBS-T $(0.1 \%$ Tween-20) was used for blocking at room temperature for $1 \mathrm{hr}$. Primary antibodies were used overnight at $4^{\circ} \mathrm{C}$ as follows: rabbit anti-PTTG1 (Abcam, Cat. \#ab79546, 1:5000), mouse anti-alpha Tubulin (Merck Millipore, Cat. \#CP06, 1:2000), Signal was detected by SuperSignal West Dura (Life Technologies, Cat. \#34075) with Otimax 2010 X-Ray Film Processor (PROTECT) and the result was quantified using ImageJ, with unpaired t-test as a statistical analysis.

\section{SAMPLE PREPARATION FOR CONFOCAL MICROSCOPY}

\section{Immunofluorescence on sections}

Standard immunofluorescence was performed on cryo- and paraffin sections. For cryosections, PFA fixed embryos were infiltrated with $15 \%$ and $30 \%$ sucrose in PBS for up to 24 hours depending on the stage, embedded in O.C.T. (Tissue-Tek® O.C.T., Sakura Finetek, Cat. \#sa-4583) and cut into $10 \mu \mathrm{m}$ coronal sections. For paraffin sections PFA fixed embryos were dehydrated, incubated in RotiHistol (Carl Roth, Cat. \#6640.1), embedded in paraffin and cut into $10 \mu \mathrm{m}$ sections. Standard immunohistochemical analysis was carried out by incubation of tissue sections with primary antibodies at the following dilutions: rabbit anti-SHH (Santa Cruz, Cat. \#sc9024, 1:50), sheep anti-LRP2 antiserum (1:4000) kindly provided by the Laboratory 
of Renata Kozyraki, rabbit anti-LRP2 (Abcam, Cat. \#ab76969, 1:1000), rabbit antiPTTG1 (Abcam, Cat. \#ab79546, 1:100). Bound primary antibodies were visualized using secondary antisera conjugated with Alexa Fluor 488, 555, 647 (1:500, Abcam). All samples were counterstained with DAPI (Invitrogen, Cat. \#62248. Sections were mounted with Dako Fluorescence Mounting Medium (Agilent, Cat. \#S302380-2).

\section{Immunocytochemistry}

For immunocytochemistry NIH-3T3 cells were seeded in chamber slides (Sigma, Cat. \#C6807) in regular 10\%FCS/DMEM medium conditions. After $24 \mathrm{~h}$ cells were rinsed with PBS(1x) fixed with 4\% PFA for 15 min, RT and permeabilized with PBS-TritonX $0.25 \%$ for $20 \mathrm{~min}$. Blocking with $10 \%$ donkey serum/PBS-TritonX for $1 \mathrm{hr}$, was followed by a standard incubation with primary and secondary antibodies as described above, with dilutions as follows: rabbit anti-PTTG1 (Abcam, Cat. \#ab79546, 1:100), goat antiPTTG1 (LifeSpan Biosciences, Cat. \#LS-B5119-50, 1:100), mouse anti-ARL13b (UC Davis/NIH NeuroMab, Cat. \#75-287, 1:500), rabbit anti-ARL13b (ProteinTech, Cat. \#17711-1-AP, 1:1500), mouse anti-acetylated Tubulin (Sigma, Cat. \#T7451, 1:1000), mouse anti-yTubulin (Sigma, Cat. \#T6557, 1:200). Walls from the chambers were disassembled according to manufacturer's instruction and samples were mounted with Dako Fluorescence mounting medium. Each experiment was repeated at least five times $n$ triplicates.

\section{Preparation of mouse cephalic explants for immunofluorescence analysis}

Explants were prepared analogically as described in (Echevarria et al., 2002). E9.5 mouse embryos were collected and the neural tube was cut open along the dorsal midline, from caudal to rostral direction, using an insect needle. The neural folds were precisely cut above the heart and placed on the sterile filter (Millipore, Cat. \#MCEP06H48) on the petri dish in the drop of PBS(1x). The floor plate at the level of the cephalic flexure was pinched in order to unfold the tissue with the ventricular part 
facing up. Filter was placed in the 6-well plate containing DMEM/10\% FCS and explants were incubated at $37^{\circ} \mathrm{C}$, with $5 \% \mathrm{CO} 2$ and $95 \%$ humidity for $3-4$ hours to flatten and recover. The explants were washed gently in PBS(1x), fixed $1 \mathrm{hr}$ in $4 \%$ PFA, and subjected to the standard immunofluorescence protocol described above, with $2 x \mathrm{O} / \mathrm{N}$ for primary antibody incubation, in the dilutions as follows: rabbit antiPTTG1 (Abcam, Cat. \#ab79546, 1:100), goat anti-PTTG1 (LifeSpan Biosciences, Cat. \#LS-B5119-50, 1:100), mouse anti-ARL13b (UC Davis/NIH NeuroMab, Cat. \#75-287, 1:500). Secondary antibodies conjugated with Alexa Fluor 488 were used to visualize primary cilia and with Alexa Fluor 647 were used to visualize PTTG1 signals. Explants were flat-mounted in Slow Fade Diamond Antifade Mountant (Invitrogen, Cat. \#S36963) using Secure-Seal Spacer (Invitrogen, Cat. \#S24737) in order to match the refractive index $(\mathrm{RI})$ of the mountant and the immersion media of the glycerol objective, and to prevent distortions of the explant by pressure, shrinking and hardening of the mountant.

\section{CONFOCAL MICROSCOPY IMAGE AQUISITION}

Image acquisitions of tissue sections were carried out using either a Leica SPE or Leica TCS SP8 confocal microscope using a HC PI Apo 20× NA 0.75 MultilMM and HC PI Apo 63× NA 1.3 oil immersion objective. All samples that were compared either for qualitative or quantitative analysis were imaged under identical settings for laser power, detector and pixel size. Cells were imaged with a Leica TCS SP5 confocal microscope equipped with a ACS Apo 63× oil NA 1.3 immersion objective.

The forebrain region of the explant samples was imaged en face with a Leica TCS SP8 confocal microscope using a HC PI Apo 63× NA 1.3 glycerol immersion objective with a WD of $0.3 \mathrm{~mm}$ to enable high-resolution imaging with minimal spherical aberrations of the thick specimen. Three regions per explants were imaged for $n=6$ C57BL/6N and $\mathrm{n}=6 \mathrm{FVB} / \mathrm{N}$. High-resolution z-stack ( $60 \mathrm{~nm}$ pixel size, 12 bit, $0.2 \mu \mathrm{m}$ z-step size) 
images of apical side of neuroepithelium were acquired with a z-piezo stepper. In all samples, Alexa Fluor 488 was excited by a $488 \mathrm{~nm}$ laser, detection at $500-550 \mathrm{~nm}$, Alexa Fluor 555 was excited by a 555 nm laser, detection at 570 - 620 nm, Alexa Fluor 647 was excited by a $633 \mathrm{~nm}$ or $647 \mathrm{~nm}$ laser, detection at $660-730 \mathrm{~nm}$ and DAPI was excited at $405 \mathrm{~nm}$, detection at $420-450 \mathrm{~nm}$ with a pinhole set to $1 \mathrm{AU}$.

\section{IMAGE PROCESSING AND ANALYSIS}

\section{D Data processing, deconvolution and correction}

Confocal Z- stacks of explants were subjected to a background correction and processed by deconvolution with the CMLE algorithm in order to obtain an improved signal to noise ratio and axial and spatial resolution using Huygens Professional 19.10 software (Scientific Volume Imaging). For optimal deconvolution the experimental PSF was calculated with Huygens PSF Distiller by using sub-resolution TetraSpeck ${ }^{\mathrm{TM}}$ Microspheres, $0.2 \mu \mathrm{m}$ (Invitrogen, Cat. \#T7280) embedded and acquired with same imaging conditions as the explant sample. The same deconvolved beads sample was used to estimate a chromatic aberration correction matrix (in $x / y / z$ ) which was used to correct the experimental sample data. To get isotropic voxel values the aspect ratio of the deconvolved bead sample was changed. This new sampling value was applied to all image data with the IMARIS Software before doing further segmentation and analysis steps.

\section{Immunofluorescence signal localization analysis}

Localization of the protein of interest (PTTG1) within the primary cilium of mouse neuroepithelium was assessed using IMARIS software (Imaris 9.3 and 9.5, Bitplane) after applying raw data corrections (deconvolution, chromatic shift correction, nonisotropic imaging correction). 3D rendering and surfaces reconstruction of individual cilia was performed for two channels - one for cilia marker (ARL13b) and one for PTTG1 - and the classical object-based colocalization approach (individual 
segmentation and voxel based colocalization) with IMARIS XT module was used. To visualize the precise spatial coexistence of the two proteins in individual cilia, views from different angles were created and transparent LUTs as well as clipping planes were used to cut the 3D surfaces open to enable seeing inside cilia. Total number of cilia in each sample was assessed during the same processing steps from the reconstructed surface of $A R L 13 b$ signal, and was given as number of cilia per area (Figure S6a).

\section{Quantification of PTTG1 immunofluorescence signal intensity}

Z-stack images of the coronal sections were analyzed using ImageJ (Fiji, NIH). For the quantification of PTTG1 signals in the neuroepithelium the full Z-stack was used. Region of interest (ROI) was manually outlined as shown in the (Figure S5d) and the mean fluorescent intensity was measured with the ROI manager. The average intensity for each animal was used in the final quantification and unpaired t-test statistical analysis was performed to assess the significance.

\section{Immunofluorescence analysis of primary cilia using STED microscopy imaging}

En face single-color stimulated emission depletion (STED) microscopy imaging was performed on the mouse cephalic explants, which were prepared as described above, with exception to the highly cross-absorbed secondary antibody Alexa Fluor Plus 594 (Invitrogen, Cat. \#A32744) and ProLong Gold Antifade mountant (Invitrogen, Cat. \#P36934) that were used to obtain optimal resolution. STED microscopy images were taken with a Leica SP8 TCS STED microscope (Leica Microsystems) equipped with a pulsed white-light excitation laser (WLL; 80 ps pulse width, $80 \mathrm{MHz}$ repetition rate (NKT Photonics) and two STED laser for depletion at $592 \mathrm{~nm}$ and $775 \mathrm{~nm}$. The system was controlled by the Leica LAS $X$ software. Single-color STED imaging was performed by exciting Alexa Fluor Plus 594 at $594 \mathrm{~nm}$ and the depletion of its emission 
with the $775 \mathrm{~nm}$ STED laser. Time gated detection was set from $0.3-6 \mathrm{~ns}$. The fluorescence signal was detected from $604-650 \mathrm{~nm}$ by a hybrid detector ( $\mathrm{HyD}$ ) at appropriate spectral regions separated from the STED laser. Images were acquired with a HC PL APO CS2 100×/1.40 NA oil objective (Leica Microsystems), a scanning format of 1,024 × 1,024 pixels, 8-bit sampling, 16x line averaging and 6x optical zoom, yielding a pixel size of $18.9 \times 18.9 \mathrm{~nm}$. Additionally, to every STED image, a confocal image with the same settings but just $1 x$ line averaging was acquired.

All STED microscope images of the cilia within each independent experiment were acquired with equal settings. Single optical plane images were subjected to the cilia length quantification in ImageJ. Regions of interest (ROIs) were manually selected with the segmented line tool and the total lengths of single cilia were measured. The average cilia lengths for both mouse lines were checked for the statistical significance using unpaired t-test.

\section{Scanning electron microscopy}

For scanning electron microscopy E8.5 embryos were dissected and fixed in $0.1 \mathrm{M}$ sodium cacodylate buffer $(\mathrm{pH} 7.3 / 7.4)$ containing $2.5 \%$ glutaraldehyde. After rinsing in cacodylate buffer a postfixation step in $2 \% \mathrm{OsO}_{4}$ for two hours was followed. Samples were dehydrated in graded ethanol series, osmicated, dried in critical point apparatus Polaron 3000, coated with gold/palladium MED 020 (BAL-TEC) and examined at the Zeiss scanning electron microscope (Gemini DSM 982).

\section{Transmission electron microscopy and immunogold labeling}

The head region of mouse embryos at E9.5 was fixed with 3\% freshly prepared formaldehyde/ $0.05 \%$ glutaraldehyde (EM-grade) in $0.1 \mathrm{M}$ phosphate buffer for 1 hour at RT. After washing, samples were infiltrated with $2.3 \mathrm{M}$ sucrose over night at $4^{\circ} \mathrm{C}$ and frozen. Semithin sections were prepared to identify the region of the neuroepithelium. Ultrathin cryosections according to Tokuyasu were labeled with anti-PTTG1 antibody 
(Abcam, Cat. \#ab79546, diluted 1:50) and $12 \mathrm{~nm}$ colloidal gold anti-rabbit secondary antibody (Dianova, Cat. \#111-205-144). Sections were contrasted and stabilized with a mixture of $3 \%$ tungstosilicic acid hydrate and $2.5 \%$ polyvinyl alcohol. EM pictures were taken at $80 \mathrm{kV}$ with a Morgagni electron microscope (Thermo Fisher), equipped with a Morada camera and the iTEM software (EMSIS GmbH, Münster, Germany).

\section{Quantification and statistical analysis}

Tests used to analyze the data were done using Prism 7 software (GraphPad) and are mentioned in the respective Figure legends. Figures were prepared using Adobe Illustrator 2019 software.

The term significant was used, if the $p$ value of a result was smaller than 0.05 . Exact $\mathrm{p}$ values, $\mathrm{n}$ numbers and biological replicates are reported in the Figure legends, supplementary tables, or according methods. 


\section{REFERENCES}

Anderson, R.M., Lawrence, A.R., Stottmann, R.W., Bachiller, D., and Klingensmith, J. (2002). Chordin and noggin promote organizing centers of forebrain development in the mouse. Development 129, 4975-4987.

Baardman, M.E., Zwier, M.V., Wisse, L.J., Groot, A.C.G., KerstjensFrederikse, W.S., Hofstra, R.M.W., Jurdzinski, A., Hierck, B.P., Jongbloed, M.R.M., Berger, R.M.F., et al. (2016). Common arterial trunk and ventricular non-compaction in Lrp2 knockout mice indicate a crucial role of LRP2 in cardiac development. Dis. Model. Mech. 9, 413-425.

Banting, G.S., Barak, O., Ames, T.M., Burnham, A.C., Kardel, M.D., Cooch, N.S., Davidson, C.E., Godbout, R., McDermid, H.E., and Shiekhattar, R. (2005). CECR2, a protein involved in neurulation, forms a novel chromatin remodeling complex with SNF2L. Hum. Mol. Genet. 14, 513-524.

Bernal, J.A., Luna, R., Espina, Á., Lázaro, I., Ramos-Morales, F., Romero, F., Arias, C., Silva, A., Tortolero, M., and Pintor-Toro, J.A. (2002). Human securin interacts with p53 and modulates p53-mediated transcriptional activity and apoptosis. Nat. Genet. 32, 306-311.

Boelaert, K., Tannahill, L.A., Bulmer, J.N., Kachilele, S., Chan, S.Y., Kim, D., Gittoes, N.J.L., Franklyn, J.A., Kilby, M.D., and Mccabe, C.J. (2003). A potential role for PTTG/securin in the developing human fetal brain. FASEB J. 17, 1631-1639.

Breslow, D.K., Hoogendoorn, S., Kopp, A.R., Morgens, D.W., Vu, B.K., Kennedy, M.C., Han, K., Li, A., Hess, G.T., Bassik, M.C., et al. (2018). A CRISPR-based screen for Hedgehog signaling provides insights into ciliary function and ciliopathies. Nat. Genet. 50, 460-471.

Chiang, C., Litingtung, Y., Lee, E., Young, K.E., Corden, J.L., Westphal, H., and Beachy, P.A. (1996). Cyclopia and defective axial patterning in mice lacking Sonic hedgehog gene function. Nature 383, 407-413.

Chih, B., Liu, P., Chinn, Y., Chalouni, C., Komuves, L.G., Hass, P.E., Sandoval, W., and Peterson, A.S. (2012). A ciliopathy complex at the transition zone protects the cilia as a privileged membrane domain. Nat. Cell Biol. 14, 61-72.

Christ, A., Christa, A., Kur, E., Lioubinski, O., Bachmann, S., Willnow, T.E., and Hammes, A. (2012). LRP2 Is an Auxiliary SHH Receptor Required to Condition the Forebrain Ventral Midline for Inductive Signals. Dev. Cell 22, 268-278.

Christ, A., Marczenke, M., and Willnow, T.E. (2020). LRP2 controls sonic hedgehog-dependent differentiation of cardiac progenitor cells during outflow tract formation. Hum. Mol. Genet. 
Cole, F., and Krauss, R.S. (2003). Microform holoprosencephaly in mice that lack the Ig superfamily member Cdon. Curr. Biol. CB 13, 411-415.

van Dam, T.J., Wheway, G., Slaats, G.G., Huynen, M.A., Giles, R.H., and SYSCILIA Study Group (2013). The SYSCILIA gold standard (SCGSv1) of known ciliary components and its applications within a systems biology consortium. Cilia 2, 7.

Davidson, C.E., Li, Q., Churchill, G.A., Osborne, L.R., and McDermid, H.E. (2007). Modifier locus for exencephaly in Cecr2 mutant mice is syntenic to the 10 q25.3 region associated with neural tube defects in humans. Physiol. Genomics 31, 244-251.

Dubourg, C., Kim, A., Watrin, E., Tayrac, M. de, Odent, S., David, V., and Dupé, V. (2018). Recent advances in understanding inheritance of holoprosencephaly. Am. J. Med. Genet. C Semin. Med. Genet. 178, 258-269.

Echevarria, D., Vieira, C., and Martinez, S. (2002). Mammalian neural tube grafting experiments: an in vitro system for mouse experimental embryology. Int. J. Dev. Biol. 45, 895-902.

Fabregat, A., Jupe, S., Matthews, L., Sidiropoulos, K., Gillespie, M., Garapati, P., Haw, R., Jassal, B., Korninger, F., May, B., et al. (2018). The Reactome Pathway Knowledgebase. Nucleic Acids Res. 46, D649-D655.

Fliegauf, M., Benzing, T., and Omran, H. (2007). When cilia go bad: cilia defects and ciliopathies. Nat. Rev. Mol. Cell Biol. 8, 880-893.

Gajera, C.R., Emich, H., Lioubinski, O., Christ, A., BeckervordersandforthBonk, R., Yoshikawa, K., Bachmann, S., Christensen, E.I., Götz, M., Kempermann, G., et al. (2010). LRP2 in ependymal cells regulates BMP signaling in the adult neurogenic niche. J. Cell Sci. 123, 1922-1930.

Garcia-Gonzalo, F.R., Corbit, K.C., Sirerol-Piquer, M.S., Ramaswami, G., Otto, E.A., Noriega, T.R., Seol, A.D., Robinson, J.F., Bennett, C.L., Josifova, D.J., et al. (2011). A transition zone complex regulates mammalian ciliogenesis and ciliary membrane composition. Nat. Genet. 43, 776-784.

Geng, X., and Oliver, G. (2009). Pathogenesis of holoprosencephaly. J. Clin. Invest. 119, 1403-1413.

Geng, X., Speirs, C., Lagutin, O., Inbal, A., Liu, W., Solnica-Krezel, L., Jeong, Y., Epstein, D.J., and Oliver, G. (2008). Haploinsufficiency of Six3 fails to activate Sonic hedgehog expression in the ventral forebrain and causes holoprosencephaly. Dev. Cell 15, 236-247.

Genkai, N., Homma, J., Sano, M., Tanaka, R., and Yamanaka, R. (2006). Increased expression of pituitary tumor-transforming gene (PTTG)- 1 is correlated with poor prognosis in glioma patients. Oncol. Rep. 15, 1569-1574.

Gerdes, J.M., Davis, E.E., and Katsanis, N. (2009). The Vertebrate Primary Cilium in Development, Homeostasis, and Disease. Cell 137, 32-45. 
Gerhardt, C., Wiegering, A., Leu, T., and Rüther, U. (2016). Control of Hedgehog Signalling by the Cilia-Regulated Proteasome. J. Dev. Biol. 4, 27.

92. Gigante, E. D. \& Caspary, T. Signaling in the primary cilium through the lens of the Hedgehog pathway. WIREs Dev. Biol. n/a, e377 (2020).

Goetz, S.C., and Anderson, K.V. (2010). The primary cilium: a signalling centre during vertebrate development. Nat. Rev. Genet. 11, 331-344.

Gromley, A., Yeaman, C., Rosa, J., Redick, S., Chen, C.-T., Mirabelle, S., Guha, M., Sillibourne, J., and Doxsey, S.J. (2005). Centriolin Anchoring of Exocyst and SNARE Complexes at the Midbody Is Required for SecretoryVesicle-Mediated Abscission. Cell 123, 75-87.

Hagting, A., den Elzen, N., Vodermaier, H.C., Waizenegger, I.C., Peters, J.M., and Pines, J. (2002). Human securin proteolysis is controlled by the spindle checkpoint and reveals when the APC/C switches from activation by Cdc20 to Cdh1. J. Cell Biol. 157, 1125-1137.

Hammes, A., Guo, J.-K., Lutsch, G., Leheste, J.-R., Landrock, D., Ziegler, U., Gubler, M.-C., and Schedl, A. (2001). Two Splice Variants of the Wilms' Tumor 1 Gene Have Distinct Functions during Sex Determination and Nephron Formation. Cell 106, 319-329.

Havens, C.G., and Walter, J.C. (2011). Mechanism of CRL4(Cdt2), a PCNAdependent E3 ubiquitin ligase. Genes Dev. 25, 1568-1582.

Hayhurst, M., and McConnell, S. (2003). Mouse models of holoprosencephaly. Curr. Opin. Neurol. 16, 135-141.

He, M., Agbu, S., and Anderson, K.V. (2017). Microtubule Motors Drive Hedgehog Signaling in Primary Cilia. Trends Cell Biol. 27, 110-125.

Hellmuth, S., Gómez-H, L., Pendás, A.M., and Stemmann, O. (2020). Securin-independent regulation of separase by checkpoint-induced shugoshin-MAD2. Nature 580, 536-541.

Heussler, H.S., Suri, M., Young, I.D., and Muenke, M. (2002). Extreme variability of expression of a Sonic Hedgehog mutation: attention difficulties and holoprosencephaly. Arch. Dis. Child. 86, 293-296.

Heyne, G.W., Everson, J.L., Ansen-Wilson, L.J., Melberg, C.G., Fink, D.M., Parins, K.F., Doroodchi, P., Ulschmid, C.M., and Lipinski, R.J. (2016). Gli2 gene-environment interactions contribute to the etiological complexity of holoprosencephaly: evidence from a mouse model. Dis. Model. Mech. 9, 1307-1315.

Holt, L.J., Krutchinsky, A.N., and Morgan, D.O. (2008). Positive feedback sharpens the anaphase switch. Nature 454, 353-357. 
Hong, M., and Krauss, R.S. (2018). Modeling the complex etiology of holoprosencephaly in mice. Am. J. Med. Genet. C Semin. Med. Genet. 178, 140-150.

Hong, M., Christ, A., Christa, A., Willnow, T.E., and Krauss, R.S. (2020). Cdon mutation and fetal alcohol converge on Nodal signaling in a mouse model of holoprosencephaly. ELife 9.

Jászai, J., Thamm, K., Karbanová, J., Janich, P., Fargeas, C.A., Huttner, W.B., and Corbeil, D. (2020). Prominins control ciliary length throughout the animal kingdom: New lessons from human prominin-1 and zebrafish prominin3. J. Biol. Chem. jbc.RA119.011253.

Jensen, A.M., and Wallace, V.A. (1997). Expression of Sonic hedgehog and its putative role as a precursor cell mitogen in the developing mouse retina. Development 124, 363-371.

Kantarci, S., Al-Gazali, L., Hill, R.S., Donnai, D., Black, G.C.M., Bieth, E., Chassaing, N., Lacombe, D., Devriendt, K., Teebi, A., et al. (2007). Mutations in LRP2, which encodes the multiligand receptor megalin, cause DonnaiBarrow and facio-oculo-acoustico-renal syndromes. Nat. Genet. 39, 957-959.

Karsten, S.L., Kudo, L.C., Jackson, R., Sabatti, C., Kornblum, H.I., and Geschwind, D.H. (2003). Global analysis of gene expression in neural progenitors reveals specific cell-cycle, signaling, and metabolic networks. Dev. Biol. 261, 165-182.

Khalifa, O., Al-Sahlawi, Z., Imtiaz, F., Ramzan, K., Allam, R., Al-Mostafa, A., Abdel-Fattah, M., Abuharb, G., Nester, M., Verloes, A., et al. (2015). Variable expression pattern in Donnai-Barrow syndrome: Report of two novel LRP2 mutations and review of the literature. Eur. J. Med. Genet. 58, 293-299.

Kim, A., Savary, C., Dubourg, C., Carré, W., Mouden, C., Hamdi-Rozé, H., Guyodo, H., Douce, J.L., Génin, E., Campion, D., et al. (2019). Integrated clinical and omics approach to rare diseases: novel genes and oligogenic inheritance in holoprosencephaly. Brain 142, 35-49.

Kim, J.C., Ou, Y.Y., Badano, J.L., Esmail, M.A., Leitch, C.C., Fiedrich, E., Beales, P.L., Archibald, J.M., Katsanis, N., Rattner, J.B., et al. (2005). MKKS/BBS6, a divergent chaperonin-like protein linked to the obesity disorder Bardet-Biedl syndrome, is a novel centrosomal component required for cytokinesis. J. Cell Sci. 118, 1007-1020.

Kooistra, M.K., Leduc, R.Y.M., Dawe, C.E., Fairbridge, N.A., Rasmussen, J., Man, J.H.Y., Bujold, M., Juriloff, D., King-Jones, K., and McDermid, H.E. (2011). Strain-specific modifier genes of Cecr2-associated exencephaly in mice: genetic analysis and identification of differentially expressed candidate genes. Physiol. Genomics 44, 35-46.

Krauss, R.S. (2007). Holoprosencephaly: new models, new insights. Expert Rev. Mol. Med. 9, 1-17. 
Krauss, R.S., and Hong, M. (2016). Chapter Thirty-Three - GeneEnvironment Interactions and the Etiology of Birth Defects. In Current Topics in Developmental Biology, P.M. Wassarman, ed. (Academic Press), pp. 569580 .

Lang, B., Pu, J., Hunter, I., Liu, M., Martin-Granados, C., Reilly, T.J., Gao, G.D., Guan, Z.-L., Li, W.-D., Shi, Y.-Y., et al. (2014). Recurrent deletions of ULK4 in schizophrenia: a gene crucial for neuritogenesis and neuronal motility. J. Cell Sci. 127, 630-640.

Lang, B., Zhang, L., Jiang, G., Hu, L., Lan, W., Zhao, L., Hunter, I., Pruski, M., Song, N.-N., Huang, Y., et al. (2016). Control of cortex development by ULK4, a rare risk gene for mental disorders including schizophrenia. Sci. Rep. 6, 31126.

Leduc, R.Y.M., Singh, P., and McDermid, H.E. (2017). Genetic backgrounds and modifier genes of NTD mouse models: An opportunity for greater understanding of the multifactorial etiology of neural tube defects. Birth Defects Res. 109, 140-152.

Li, Y., Klena, N.T., Gabriel, G.C., Liu, X., Kim, A.J., Lemke, K., Chen, Y., Chatterjee, B., Devine, W., Damerla, R.R., et al. (2015). Global genetic analysis in mice unveils central role for cilia in congenital heart disease. Nature 521, 520-524.

Liu, M., Guan, Z., Shen, Q., Lalor, P., Fitzgerald, U., O’Brien, T., Dockery, P., and Shen, S. (2016). Ulk4 Is Essential for Ciliogenesis and CSF Flow. J. Neurosci. Off. J. Soc. Neurosci. 36, 7589-7600.

Longoni, M., Kantarci, S., Donnai, D., and Pober, B.R. (1993). Donnai-Barrow Syndrome. 2008 Aug 28 [Update 2018 Nov 21]. In GeneReviews®, M.P. Adam, H.H. Ardinger, R.A. Pagon, S.E. Wallace, L.J. Bean, K. Stephens, and A. Amemiya, eds. (Seattle (WA): University of Washington, Seattle); 19932019.

Love, M.I., Huber, W., and Anders, S. (2014). Moderated estimation of fold change and dispersion for RNA-seq data with DESeq2. Genome Biol. 15, 550 .

Mei, J., Huang, X., and Zhang, P. (2001). Securin is not required for cellular viability, but is required for normal growth of mouse embryonic fibroblasts. Curr. Biol. CB 11, 1197-1201.

Ming, J.E., and Muenke, M. (2002). Multiple Hits during Early Embryonic Development: Digenic Diseases and Holoprosencephaly. Am. J. Hum. Genet. 71, 1017-1032.

Mirandola, L., Figueroa, J.A., Phan, T.T., Grizzi, F., Kim, M., Rahman, R.L., Jenkins, M.R., Cobos, E., Jumper, C., Alalawi, R., et al. (2015). Novel antigens in non-small cell lung cancer: SP17, AKAP4, and PTTG1 are potential immunotherapeutic targets. Oncotarget 6, 2812-2826. 
Moreno-Mateos, M.A., Espina, Á.G., Torres, B., del Estal, M.M.G., RomeroFranco, A., Ríos, R.M., and Pintor-Toro, J.A. (2011). PTTG1/securin modulates microtubule nucleation and cell migration. Mol. Biol. Cell 22, 43024311.

Muenke, M., and Beachy, P.A. (2000). Genetics of ventral forebrain development and holoprosencephaly. Curr. Opin. Genet. Dev. 10, 262-269.

Muenke, M., and Cohen, M.M. (2000). Genetic approaches to understanding brain development: Holoprosencephaly as a model. Ment. Retard. Dev. Disabil. Res. Rev. 6, 15-21.

Nachury, M.V., and Mick, D.U. (2019). Establishing and regulating the composition of cilia for signal transduction. Nat. Rev. Mol. Cell Biol. 20, 389405.

Ozdemir, H., Plamondon, J., Gaskin, P., Asoglu, M.R., and Turan, S. (2019). A prenatally diagnosed case of Donnai-Barrow syndrome: Highlighting the importance of whole exome sequencing in cases of consanguinity. Am. J. Med. Genet. A.

Pan, J., You, Y., Huang, T., and Brody, S.L. (2007). RhoA-mediated apical actin enrichment is required for ciliogenesis and promoted by Foxj1. J. Cell Sci. 120, 1868-1876.

Park, T.J., Mitchell, B.J., Abitua, P.B., Kintner, C., and Wallingford, J.B. (2008). Dishevelled controls apical docking and planar polarization of basal bodies in ciliated epithelial cells. Nat. Genet. 40, 871-879.

Petryk, A., Anderson, R.M., Jarcho, M.P., Leaf, I., Carlson, C.S., Klingensmith, J., Shawlot, W., and O'Connor, M.B. (2004). The mammalian twisted gastrulation gene functions in foregut and craniofacial development. Dev. Biol. 267, 374-386.

Pober, B.R., Longoni, M., and Noonan, K.M. (2009). A review of DonnaiBarrow and facio-oculo-acoustico-renal (DB/FOAR) syndrome: Clinical features and differential diagnosis. Birt. Defects Res. A. Clin. Mol. Teratol. 85, 76-81.

Pusapati, G.V., Kong, J.H., Patel, B.B., Krishnan, A., Sagner, A., Kinnebrew, M., Briscoe, J., Aravind, L., and Rohatgi, R. (2018). CRISPR Screens Uncover Genes that Regulate Target Cell Sensitivity to the Morphogen Sonic Hedgehog. Dev. Cell 44, 113-129.e8.

Read, M.L., Fong, J.C., Modasia, B., Fletcher, A., Imruetaicharoenchoke, W., Thompson, R.J., Nieto, H., Reynolds, J.J., Bacon, A., Mallick, U., et al. (2017). Elevated PTTG and PBF predicts poor patient outcome and modulates DNA damage response genes in thyroid cancer. Oncogene 36, 5296-5308.

Roessler, E., and Muenke, M. (2010). The molecular genetics of holoprosencephaly. Am. J. Med. Genet. C Semin. Med. Genet. 154C, 52-61. 
Roessler, E., Belloni, E., Gaudenz, K., Jay, P., Berta, P., Scherer, S.W., Tsui, L.-C., and Muenke, M. (1996). Mutations in the human Sonic Hedgehog gene cause holoprosencephaly. Nat. Genet. 14, 357.

Rosenfeld, J.A., Ballif, B.C., Martin, D.M., Aylsworth, A.S., Bejjani, B.A., Torchia, B.S., and Shaffer, L.G. (2010). Clinical characterization of individuals with deletions of genes in holoprosencephaly pathways by aCGH refines the phenotypic spectrum of HPE. Hum. Genet. 127, 421-440.

Rothfels, K. (2014). Hedgehog "off" State. Reactome - Curated Knowledgebase Biol. Pathw. 50.

Sabatino, J.A., Stokes, B.A., and Zohn, I.E. (2017). Prevention of neural tube defects in Lrp2 mutant mouse embryos by folic acid supplementation. Birth Defects Res. 109, 16-26.

Salehi, F., Scheithauer, B.W., Sharma, S., Kovacs, K., Lloyd, R.V., Cusimano, M.D., and Munoz, D.G. (2013). Immunohistochemical expression of PTTG in brain tumors. Anticancer Res. 33, 119-122.

Sasaki, H., Hui, C., Nakafuku, M., and Kondoh, H. (1997). A binding site for Gli proteins is essential for HNF-3beta floor plate enhancer activity in transgenics and can respond to Shh in vitro. Development 124, 1313-1322.

Schachter, K.A., and Krauss, R.S. (2008). Chapter 3 Murine Models of Holoprosencephaly. In Current Topics in Developmental Biology, (Academic Press), pp. 139-170.

Shah, A.S., Farmen, S.L., Moninger, T.O., Businga, T.R., Andrews, M.P., Bugge, K., Searby, C.C., Nishimura, D., Brogden, K.A., Kline, J.N., et al. (2008). Loss of Bardet-Biedl syndrome proteins alters the morphology and function of motile cilia in airway epithelia. Proc. Natl. Acad. Sci. 105, 33803385.

Sharma, N., Kosan, Z.A., Stallworth, J.E., Berbari, N.F., and Yoder, B.K. (2011). Soluble levels of cytosolic tubulin regulate ciliary length control. Mol. Biol. Cell 22, 806-816.

Singla, V., and Reiter, J.F. (2006). The Primary Cilium as the Cell's Antenna: Signaling at a Sensory Organelle. Science 313, 629-633.

Smith, K.R., Kieserman, E.K., Wang, P.I., Basten, S.G., Giles, R.H., Marcotte, E.M., and Wallingford, J.B. (2011). A role for central spindle proteins in cilia structure and function. Cytoskeleton 68, 112-124.

Solomon, B.D., Mercier, S., Vélez, J.I., Pineda-Alvarez, D.E., Wyllie, A., Zhou, N., Dubourg, C., David, V., Odent, S., Roessler, E., et al. (2010). Analysis of genotype-phenotype correlations in human holoprosencephaly. Am. J. Med. Genet. C Semin. Med. Genet. 154C, 133-141.

Spektor, A., Tsang, W.Y., Khoo, D., and Dynlacht, B.D. (2007). Cep97 and CP110 Suppress a Cilia Assembly Program. Cell 130, 678-690. 
Spoelgen, R., Hammes, A., Anzenberger, U., Zechner, D., Andersen, O.M., Jerchow, B., and Willnow, T.E. (2005). LRP2/megalin is required for patterning of the ventral telencephalon. Development 132, 405-414.

Taipale, J., Chen, J.K., Cooper, M.K., Wang, B., Mann, R.K., Milenkovic, L., Scott, M.P., and Beachy, P.A. (2000). Effects of oncogenic mutations in Smoothened and Patched can be reversed by cyclopamine. Nature 406 , 1005.

Tarabykin, V., Britanova, O., Fradkov, A., Voss, A., Katz, L.S., Lukyanov, S., and Gruss, P. (2000). Expression of PTTG and prc1 genes during telencephalic neurogenesis. Mech. Dev. 92, 301-304.

Tekendo-Ngongang, C., Muenke, M., and Kruszka, P. (1993).

Holoprosencephaly Overview. 2000 Dec 27 [Updated 2020 Mar 5]. In GeneReviews ${ }^{\circledR}$, M.P. Adam, H.H. Ardinger, R.A. Pagon, S.E. Wallace, L.J. Bean, K. Stephens, and A. Amemiya, eds. (Seattle (WA): University of Washington, Seattle); 1993-2020.

Tekendo-Ngongang, C., Owosela, B., Muenke, M., and Kruszka, P. (2020). Comorbidity of congenital heart defects and holoprosencephaly is likely genetically driven and gene-specific. Am. J. Med. Genet. C Semin. Med. Genet. 184, 154-158.

Thornton, B.R., and Toczyski, D.P. (2003). Securin and B-cyclin/CDK are the only essential targets of the APC. Nat. Cell Biol. 5, 1090-1094.

Tong, Y., Ben-Shlomo, A., Zhou, C., Wawrowsky, K., and Melmed, S. (2008). Pituitary tumor transforming gene 1 regulates Aurora kinase A activity. Oncogene 27, 6385-6395.

Trapnell, C., Pachter, L., and Salzberg, S.L. (2009). TopHat: discovering splice junctions with RNA-Seq. Bioinformatics 25, 1105-1111.

Vertii, A., Bright, A., Delaval, B., Hehnly, H., and Doxsey, S. (2015). New frontiers: discovering cilia-independent functions of cilia proteins. EMBO Rep. $16,1275-1287$.

Vlotides, G., Eigler, T., and Melmed, S. (2007). Pituitary Tumor-Transforming Gene: Physiology and Implications for Tumorigenesis. Endocr. Rev. 28, 165186.

Wallis, D., and Muenke, M. (2000). Mutations in holoprosencephaly. Hum. Mutat. 16, 99-108.

Wallis, D.E., and Muenke, M. (1999). Molecular Mechanisms of Holoprosencephaly. Mol. Genet. Metab. 68, 126-138.

Wallis, D.E., Roessler, E., Hehr, U., Nanni, L., Wiltshire, T., Richieri-Costa, A., Gillessen-Kaesbach, G., Zackai, E.H., Rommens, J., and Muenke, M. (1999). Mutations in the homeodomain of the human SIX3 gene cause holoprosencephaly. Nat. Genet. 22, 196-198. 
Wang, L., and Dynlacht, B.D. (2018). The regulation of cilium assembly and disassembly in development and disease. Development 145.

Wang, L., Piao, T., Cao, M., Qin, T., Huang, L., Deng, H., Mao, T., and Pan, J. (2013). Flagellar regeneration requires cytoplasmic microtubule depolymerization and kinesin-13. J. Cell Sci. 126, 1531-1540.

Wang, W., Wu, T., and Kirschner, M.W. (2014). The master cell cycle regulator APC-Cdc20 regulates ciliary length and disassembly of the primary cilium. ELife 3, e03083.

Willnow, T.E., Hilpert, J., Armstrong, S.A., Rohlmann, A., Hammer, R.E., Burns, D.K., and Herz, J. (1996). Defective forebrain development in mice lacking gp330/megalin. Proc. Natl. Acad. Sci. 93, 8460-8464.

Xiang, W., Wu, X., Huang, C., Wang, M., Zhao, X., Luo, G., Li, Y., Jiang, G., Xiao, X., and Zeng, F. (2017). PTTG1 regulated by miR-146a-3p promotes bladder cancer migration, invasion, metastasis and growth. Oncotarget 8 , 664-678.

Yan, H., Wang, W., Dou, C., Tian, F., and Qi, S. (2015). Securin promotes migration and invasion via matrix metalloproteinases in glioma cells. Oncol. Lett. 9, 2895-2901.

Yanagida, M. (2000). Cell cycle mechanisms of sister chromatid separation; roles of Cut1/separin and Cut2/securin. Genes Cells Devoted Mol. Cell. Mech. $5,1-8$.

Zarbalis, K., May, S.R., Shen, Y., Ekker, M., Rubenstein, J.L.R., and Peterson, A.S. (2004). A focused and efficient genetic screening strategy in the mouse: identification of mutations that disrupt cortical development. PLoS Biol. 2, E219.

Zhang, W., Kang, J.-S., Cole, F., Yi, M.-J., and Krauss, R.S. (2006). Cdo Functions at Multiple Points in the Sonic Hedgehog Pathway, and CdoDeficient Mice Accurately Model Human Holoprosencephaly. Dev. Cell 10, 657-665.

Zheng, Y., Guo, J., Zhou, J., Lu, J., Chen, Q., Zhang, C., Qing, C., Koeffler, H.P., and Tong, Y. (2015). FoxM1 transactivates PTTG1 and promotes colorectal cancer cell migration and invasion. BMC Med. Genomics 8, 49.

Zou, H., McGarry, T.J., Bernal, T., and Kirschner, M.W. (1999). Identification of a Vertebrate Sister-Chromatid Separation Inhibitor Involved in Transformation and Tumorigenesis. Science 285, 418-422.

Zuo, X., Guo, W., and Lipschutz, J.H. (2009). The Exocyst Protein Sec10 Is Necessary for Primary Ciliogenesis and Cystogenesis In Vitro. Mol. Biol. Cell 20, 2522-2529.

Zywitza, V., Misios, A., Bunatyan, L., Willnow, T.E., and Rajewsky, N. (2018). Single-Cell Transcriptomics Characterizes Cell Types in the Subventricular 
Zone and Uncovers Molecular Defects Impairing Adult Neurogenesis. Cell Rep. 25, 2457-2469.e8. 